\title{
Studies of the Relationship Between Environmental Forcing and the Structure and Dynamics of Tornado-like Vortices
}

\author{
David S. Nolan* \\ Department of Atmospheric Science \\ Colorado State University \\ Fort Collins, Colorado
}

Ann S. Almgren, John B. Bell

Computing Sciences Division

Lawrence Berkeley National Laboratory

Berkeley, California

September 4th, 2000

*Corresponding author address: Dr. David S. Nolan, Department of Atmospheric Science, Colorado State University, Fort Collins, CO 80523; nolan@chandra.atmos.colostate.edu 


\begin{abstract}
Axisymmetric numerical simulations continue to provide insight into how the structure, dynamics, and maximum windspeeds of tornadoes, and other convectively-maintained vortices, are influenced by the surrounding environment. This work is continued with a new numerical model of axisymmetric incompresible flow that incorporates adaptive mesh refinement. The model dynamically increases or decreases the resolution in regions of interest as determined by a specified refinement criterion. Here, the criterion used is based on the cell Reynolds number $\Delta x \Delta v / v$, so that the flow is guaranteed to be laminar on the scale of the local grid spacing.

The model is used to investigate how the altitude and shape of the convective forcing, the size of the domain, and the effective Reynolds number (based on the choice of the eddy viscosity v) influence the structure and dynamics of the vortex. Over a wide variety of domain and forcing geometries, the vortex Reynolds number $\Gamma / v$ (the ratio of the far-field circulation to the eddy viscosity) is shown to be the most important parameter for determining vortex structure and behavior. Furthermore, it is found that the vertical scale of the convective forcing only affects the vortex inasmuch as this vertical scale contributes to the total strength of the convective forcing. The horizontal scale of the convective forcing, however, is found to be the fundamental length scale in the problem, in that it can determine both the circulation of the fluid that is drawn into the vortex core, and also influences the depth of the swirling boundary layer. Higher mean windspeeds are sustained as the eddy viscosity is decreased; however, it is observed that the highest windspeeds are found in the high-swirl, two-celled vortex regime rather than in the low-swirl, one-celled regime, which is contrast with some previous results.
\end{abstract}


The conclusions drawn from these results are applied to dimensional simulations with scales similar to the mesocyclone/thunderstorm environment. Tornado-like vortices are reproduced, using a constant eddy viscosity with such values as $40 \mathrm{~m}^{2} \mathrm{~s}^{-1}$, which have maximum windspeeds, radii of maximum winds, and boundary layer depths which are quite similar to those recently observed with portable Doppler radar. Based on the results of both nondimensional and tornado-scale simulations, scaling laws are empirically derived for the internal length scales in tornado-like vortices, such as the depth of the boundary layer and the radius of maximum winds. 


\section{Introduction}

\section{a. Recent results on the structure and dynamics of tornado-like vortices}

Axisymmetric modelling of the forced convergence of rotating fluid near a lower boundary has been an invaluable tool in the study of a tornado's interaction with the surface. The early models, such as Rotunno (1977,1979), Walko and Gall (1986), and Howells et al. (1988) were designed to represent axisymmetric versions of laboratory models such as those used by Ward (1972) and Church et al. (1979). In these models (physical and numerical) rotating air was fed into the lower levels of a cylindrical chamber and drawn out through the top with some kind of forcing (a fan or boundary conditions). The most important result from the laboratory studies was that the structure and behavior of the resulting vortex was well-correlated with the ratio of the circulation of the fluid entering the vortex chamber to the volume flow rate of the air through the chamber, a parameter generally known as the swirl ratio. Perhaps the most important discovery found with the numerical models, first made by Rotunno (1979) and furthered explored by Howells et al. (1988), is that the near-surface windspeeds are substantially higher when no-slip boundary conditions are used at the surface. This observation identifies the importance of radial inflow in the swirling boundary layer which amplifies the convergence of the rotating fluid just above the boundary layer.

Rather than using a domain based on laboratory models, Fiedler (1993, 1994) (hereafter F93 and F94) simulated the formation of a vortex in a closed domain through the convergence of incompressible fluid in solid body rotation. This convergence was forced by the imposition of a fixed buoyancy function along the center axis. The integral of this vertical forcing along the center 
axis provides a velocity scale that can be considered analogous to the same velocity scale associated with the convective available potential energy (CAPE) in the atmosphere. This velocity scale provides a direct connection between the maximum windspeeds in a tornado and those observed in the closed-domain model.

Nolan and Farrell (1999a) (hereafter NF99) used a similar numerical model of axisymmetric, incompressible fluid flow in a closed domain to study tornado-like vortices. Rather than focusing on the maximum windspeeds, they examined how the structure and dynamics of such vortices depend on the parameters that arise from dimensional analysis. They found that the results were best described by two dimensionless parameters. One of these is a convective Reynolds number

$$
R e_{C}=\frac{U L}{v},
$$

where $U$, defined by

$$
\frac{U^{2}}{2}=\int_{0}^{Z_{\max }} F_{z}(0, z) d z
$$

is a velocity scale based on the integral of the convective forcing [see (3.5) below] along the center axis of the domain, $L$ is the length scale in the domain, and $v$ is the model eddy viscosity. The other dimensionless parameter is a vortex Reynolds number

$$
R e_{V}=\frac{\Gamma}{v}
$$

where $\Gamma$ is the circulation of the fluid in the far field; when the fluid is in solid body rotation at rotation rate $\Omega, \Gamma \propto \Omega L^{2}$. The way in which these parameters controlled the results could be measured both in terms of the maximum azimuthal windspeeds in the vortex core and in terms of the structure and time-dependent behavior of the flow. For example, the average maximum wind- 
speeds were found to follow the relation:

$$
V_{\max }=C_{v} U
$$

where $U$ is the convective velocity scale from (1.2) and $C_{v}$ is a velocity coefficient whose value typically lies between 0.7 and 1.0 and depends, albeit weakly, on both $\operatorname{Re}_{V}$ and $\operatorname{Re}_{C}$. Furthermore, NF99 found that there is a particular choice of $R e_{V}$ which maximizes $C_{v}$ for all values of $R e_{C}$, and this maximum value of $C_{v}$ increases with increasing $R e_{C}$. The physical interpretation of these relationships is as follows: 1) there is a universal structure for the tornado-like vortex which maximizes the azimuthal wind speeds, and 2) the windspeeds will increase as the viscosity is decreased, provided the circulation in the far-field is adjusted so as to maintain the ideal structure.

The value of $C_{v}$ is significant because one of the problems with earlier axisymmetric simulations is that they did not predict sufficiently high windpseeds. For example, with a CAPE of $2500 \mathrm{~J} \mathrm{~kg}^{-1}$ we have a convective velocity scale of $U=70.7 \mathrm{~m} \mathrm{~s}^{-1}$; for a typical value of $C_{v}=0.7$ we would have only $V_{\max }=49.5 \mathrm{~ms}^{-1}$. Values of $C_{v}$ closer to 1.0 would bring the model results closer to typically observed or estimated tornadic windspeeds of 70-80 $\mathrm{m} \mathrm{s}^{-1}$. By using a spatially varying viscosity, so that the upper levels of the domain did not require high resolution, Fiedler (1994) did observe azimuthal windspeeds which indicate $C_{v} \approx 1.1$ when the nondimensional viscosity in the boundary layer was $v=0.000125$, one quarter of the value used for most of the simulations in F93 and NF99. However, more recently reported axisymmetric simulations with similar configurations by Fiedler (1998) indicate that the long-time mean of $C_{v}$ in those cases is closer to 1.0.

The structure of the flow through the vortex core, and whether this flow is steady or unsteady, was found by NF99 to depend strongly on the vortex Reynolds number $R e_{V}$ and only very weakly on $R e_{C}$. While the laboratory modelers (Ward, 1972; Church et al., 1979) found that the structure of the vortex could be predicted by the swirl ratio, in a closed-domain model the low- 
level inflow of radial and angular momentum are not under the control of the modeler, but rather are determined by the swirling boundary layer which develops underneath the larger vortex aloft. NF99 introduced an internal swirl ratio which is analogous to the swirl ratio for laboratory experiments. This internal swirl ratio is defined using an arbitrary control volume around the intense part of the vortex to measure the ratio of the circulation entering the vortex core to the volume flow rate through the core, i.e.:

$$
S_{I}=\frac{r_{0}}{2 h_{0}} \frac{\int_{0}^{h_{0}} \Gamma^{*}\left(r_{0}, z\right) d z}{\int_{0}^{r_{0}} w\left(r, z_{0}\right) 2 \pi r d r}
$$

where $\Gamma^{*}(r, z)=2 \pi r v(r, z)$ is the circulation ${ }^{1}, v$ and $w$ are the azimuthal and vertical velocity fields, and $r_{0}$ and $h_{0}$ are the radius and height of the control volume that is adjacent to the surface and surrounds the vortex core. Despite the $r_{0} / 2 h_{0}$ prefactor held over from the original swirl ratio (see Church et al., 1979), observed numerical values of $S_{I}$ are not similar to those of the original swirl ratio; furthermore $S_{I}$ is arbitrary since it depends strongly on the choice of control volume $\left(r_{0}\right.$ and $\left.h_{0}\right)$. However, NF99 found that this definition of swirl ratio shares the same utility as its predecessor in that a correlation can be made between the structure of the tornado-like vortex and the value of $S_{I}$, such that as $S_{I}$ increases the vortex transitions from a one-celled vortex to a drowned vortex jump and ultimately to a two-celled vortex. As shown in Fig. 1, it was found that the value of $S_{I}$ was approximately a function of only $\Omega / \nu$ for a wide range of parameter space, thus showing how the structure of the flow in the vortex core is a function of $\operatorname{Re}_{V}$ but not of $\operatorname{Re}_{C}$ (when the length scale is fixed, $\Gamma \propto \Omega$ ). The unsteadiness of the flow in the core, as measured by

1. A factor of $2 \pi$ is included in the circulation which appears in the swirl ratio. Elsewhere it is neglected. 
the variance of $S_{I}$ (not shown - see NF99), was nearly zero for $R e_{V}<250$, but then rapidly increased for $R e_{V}>250$. As can be seen from the lower-right hand part of Fig. 1, the accuracy of the relationship $S_{I}=f\left(R e_{V}\right)$ appears to break down in this region. This unsteadiness was shown to be caused by axisymmetric disturbances propagating down into the vortex from the upper part of the domain; such downward propagation is allowed to occur by both a decrease in the vertical velocities and an increase in the gradients of the azimuthal winds in the vortex core.

Despite its utility, the internal swirl ratio suffers from some flaws. First, its definition is quite arbitrary and its value depends strongly on the size and shape of the control volume. Second, we have observed in highly unsteady simulations (usually associated with a two-celled vortex) that occasionally there can be a net flow reversal in the vortex core, such that the internal swirl ratio becomes negative or undefined. An alternative measure of the structure of the vortex is the vortex aspect ratio, defined as the ratio of the radius of maximum azimuthal winds (RMW) to the altitude of maximum azimuthal winds (ZMW):

$$
A_{V}=\frac{\mathrm{RMW}}{\mathrm{ZMW}} .
$$

Fig. 2 shows the mean value of $A_{V}$ as a function of $\Omega$ and $v$ for the same ensemble of simulations as those used to produce Fig. 1 . While $A_{V}$ is not quite as well matched with $\Omega / \nu$ as is $S_{I}$, there is still a meaningful correlation. It is interesting to note that the correlation is the strongest for large $R e_{V}$, which is exactly where $S_{I}$ has the worst correlation, indicating that $A_{V}$ is a more reliable measure of the vortex structure in the unsteady flow regime. Furthermore, $A_{V}$ does not become undefined during flow reversals, and unlike $S_{I}$ it can be measured directly with Doppler radar [see, for example, the observations of Wurman et al., (1996) where the location of the maximum azimuthal winds is easily identified]. 


\section{b. A general paradigm for intense atmospheric vortices}

The term "tornado-like vortex" was used by Fiedler (1993) to describe the crude representation of a tornado that can be generated in a simple numerical model. The model vortex, and tornadoes, share the fundamental elements necessary to produce an intense, vertically oriented vortex at the surface: rotating fluid in contact with the surface, forced to converge at low levels by overhead convection. These basic elements are also the necessary ingredients for the formation of other intense atmospheric vortices of different scales: the dust devil, which occurs when low-level rotation interacts with a developing thermal (Sinclair, 1969, 1973); waterspouts, which are believed to occur when a cumulus tower updraft interacts with a low-level shear zone (Golden, 1974a, 1974b; Simpson et al., 1986; Golden and Bluestein, 1993); and non-mesocyclone tornadoes, which are dynamically equivalent to waterspouts (hence the nickname "landspouts") (Wakimoto and Wilson, 1989; Brady and Szoke, 1989; Lee and Wilhelmson, 1997a, 1997b).

The term "tornado-like" vortex can be expanded to include all vortices which are created by the forced convergence of low-level rotation - whether in the atmosphere, in the laboratory, or in numerical models. From visual observation, dust devils, waterspouts, and tornadoes have remarkable dynamical similarity, especially in regards to their interaction with the surface (their swirling boundary layers). Such observations are further supported by the strong similarities in the structure of vortices produced in laboratory models, laboratory-analog numerical models, and closed-domain numerical models. These facts lead us to believe that the details of the source of rotation (vertical vorticity) and the source of convection (vertical motion) are not important to the dynamics of these intense vortices - particularly in regards to their surface interaction.

Which leads us to the question: which properties of the convective and rotational forcings are important to the tornado-like vortex? As outlined above, the work of Fiedler (1993, 1994, 
1998), Trapp and Davies-Jones (1997), and NF99 have begun to answer some of these questions. However, some seemingly relevant issues have not been addressed in these previous studies. Does the vertical extent of the domain, and in particular the interaction of the convective outflow with an upper boundary (tropopause), have an impact on the low-level vortex? Do the size and shape of the convective forcing play a role? Also, what determines the length scales in the tornado-like vortex (i.e., the radius of maximum winds, the depth of the boundary layer)? Can they be predicted from the environment?

In this report we investigate how the geometries of both the model domain and the vertical forcing field affect the structure, dynamics, and length scales of tornado-like vortices. We also further explore the relationships found by NF99 by investigating the dynamics of tornado-like vortices over a wider range of parameters. Section 2 discusses our numerical model which uses the feature of adaptive mesh refinement so that high Reynolds number simulations in large domains can be performed accurately and efficiently. In section 3 the model results are validated by comparison to equivalent full-resolution simulations. In section 4 we investigate how the size of the domain, the location of the forcing field, and its geometry affect the vortex dynamics. In section 5 we show the results of simulations with higher Reynolds numbers. Section 6 presents the results of simulations with dimensional scales chosen to reproduce tornado-like vortices with similar length and velocity scales as those observed in the atmosphere. In Section 7 we discuss how these length scales can be predicted. Conclusions are drawn in section 8.

\section{Numerical Modelling of Tornado-Like Vortices and the Use of Adaptive Mesh Refinement}

In past and recent years, two approaches have been taken to mitigate the computational demands of simulating tornado-like vortices. The first, which was widely used in early numerical 
simulations, is to restrict the domain to the point where only the most intense part of the vortex is resolved: the boundary layer, the "corner" region near the surface, and the vortex breakdown immediately aloft. Such models were essentially numerical analogues of the early laboratory models, which fed swirling air in through an opening in the sides of the domain and drew air out through an opening at top of the domain (Rotunno, 1977, 1979; Walko and Gall, 1984; Howells et al., 1988). The second approach is the use of stretched grids. For the tornado problem, the grids have been stretched so that the grid spacing decreases near the center axis and near the surface, with the highest density of grid points occurring in the corner region near $r=0, z=0$. This method has been used in both two- and three-dimensional models by Fiedler (1993, 1994, 1998), Trapp and Fiedler (1995), and Trapp and Davies-Jones (1997) so that a much larger domain could be used while still resolving the boundary layer. Both domain restriction and grid stretching have been used by W. Lewellen et al. (1997) and D. Lewellen et al. (2000) in their Large-Eddy-Simulations of the corner region.

For the purposes of this study, whose main goal is to elucidate the relationships between the structure and dynamics of the tornado-like vortex and the larger environment, restriction of the domain to the corner flow region cannot be used. Such domain restriction, by construction, decouples the vortex from the surrounding environment, and must assume predetermined properties for the inflow and outflow of the corner region. As Lewellen et al. (2000) showed in some detail, changes in the inflow and outflow boundary conditions have significant impacts on the near-surface vortex structure. Similar conclusions were found by Smith (1987) and Fiedler (1995). Thus the logical choice for this study is the use of a larger, closed domain.

While stretched grids offer many advantages over regularly spaced grids, they also have some limitations. In particular, stretched grids such as those used by Fiedler $(1993,1994,1998)$ 
and Trapp and Davies-Jones (1997) are focused on only one region of dynamical interest and are fixed in time. Trapp and Fiedler (1995) used a stretched grid which evolved in time, but could only focus on one region of interest at each moment. Thus, one cannot be assured that the flow is sufficiently resolved in all parts of the domain at all times. Furthermore, to maintain numerical stability, stretched grids require higher dissipation (viscosity) in the parts of the domain with larger grid spacing, which may have some effect on the dynamics. For example, viscosity which increases with height (to account for decreasing resolution) could have an effect on the location and character of the vortex breakdown that occurs in the tornado's outflow.

We choose instead to use the method of Adaptive Mesh Refinement (AMR). The idea of multiply nested, refined grids is not in itself new, and has been used with great success in computational fluid dynamics, including numerical simulations of supercell thunderstorms, some of which have even produced low-level vortices similar to tornadoes (Wicker and Wilhelmson, 1995; Grasso and Cotton, 1995). However, our AMR model has three additional features: 1) The locations of the refined grids are not determined intermittently by the user, but rather are controlled by an arbitrary refinement condition; 2) refined grids are added and removed automatically by the model as the flow evolves; and 3) an unlimited (except by computational resources) number of refined grids, and also levels of refinement, can be supported by the model, i.e., the model can have several levels of refinement focused on several distinct regions of dynamical interest at the same time.

We will briefly describe the essential features of our numerical model of axisymmetric, incompressible flow with AMR. The complete details of the method and some examples of its application are described in Almgren, et al. (1998). The velocities and pressure in the equations of motion [(3.1)-(3.4) below] are solved using an approximate projection method on an adaptive 
hierarchy of rectangular grids. The term "projection" identifies the method as a two-step process: first, the equations of motion are integrated forward in time for one time step, without regard to the incompressibility constraint. Second, the change in the velocity fields is projected onto the space of divergence free fields, and only the divergence-free part is retained. The method is considered "approximate" in that the divergence of the resulting velocity field is not exactly zero, but $O\left(h^{2}\right)$, where $h$ is the grid spacing. The enforcement of the incompressibility condition in approximate projection methods can generally be performed much more efficiently than in exact projection methods, with very little penalty in terms of overall accuracy.

The "adaptive hierarchy of rectangular grids" refers to a collection of two-way interacting, nested grids which are continuously updated as the flow evolves. The initial creation of the grid hierarchy, and the subsequent regridding operations, are based on refinement criteria specified by the user. In all the calculations presented here, we use as a refinement criterion the cell Reynolds number

$$
R e_{\text {cell }}=\frac{\max \{\Delta r, \Delta z\} \times \max \{\Delta u, \Delta v, \Delta w\}}{v},
$$

which is the product of the larger grid spacing and the largest velocity difference across each cell

divided by the kinematic viscosity $v . R e_{c e l l}$ is a useful measure of how well the flow is resolved by the local grid spacing, and refinement criteria on the order of $R e_{c e l l}<20$ are shown below to give good results. By using such criteria, we are assured that the flow is always laminar on the scale of the local grid spacing.

\section{Reproduction of Full-Resolution Results with Adaptive- Mesh Refinement}

In this section we use a single-grid version of the model described above to perform a 
numerical simulation of a tornado-like vortex. We then perform identical simulations with a base grid of substantially lower resolution but which use the adaptive mesh refinement capability of the model. The results show that an AMR model can accurately reproduce the results of a full-resolution model.

\section{a. Equations of motion}

We proceed directly to the non-dimensional equations of motion. For a discussion of the dimensional equations, non-dimensionalization, and the relevance of the dimensionless parameters, see NF99 and earlier references [e.g., F93, Howells et al., (1988)]. For axisymmetric, incompressible flow, the momentum equations in the radial, azimuthal, and vertical directions are, respectively,

$$
\begin{aligned}
& \frac{\partial u}{\partial t}+u \frac{\partial u}{\partial r}+w \frac{\partial u}{\partial z}-\frac{v^{2}}{r}=-\frac{\partial p}{\partial r}+v\left[\frac{\partial}{\partial r}\left(\frac{1}{r} \frac{\partial}{\partial r}(r u)\right)+\frac{\partial^{2} u}{\partial z^{2}}\right] \\
& \frac{\partial v}{\partial t}+u \frac{\partial v}{\partial r}+w \frac{\partial v}{\partial z}+\frac{u v}{r}=v\left[\frac{\partial}{\partial r}\left(\frac{1}{r} \frac{\partial}{\partial r}(r v)\right)+\frac{\partial^{2} v}{\partial z^{2}}\right] \\
& \frac{\partial w}{\partial t}+u \frac{\partial w}{\partial r}+w \frac{\partial w}{\partial z}=-\frac{\partial p}{\partial z}+v\left[\frac{1}{r} \frac{\partial}{\partial r}\left(r \frac{\partial w}{\partial r}\right)+\frac{\partial^{2} w}{\partial z^{2}}\right]+F_{z}
\end{aligned}
$$

while the incompressibility condition is

$$
\frac{1}{r} \frac{\partial}{\partial r}(r u)+\frac{\partial w}{\partial z}=0
$$

where $u$ is the radial velocity, $v$ is the azimuthal (swirling) velocity, $w$ is the vertical velocity, $p$ is the pressure, $v$ is the kinematic viscosity, and $F_{z}$ is a spatially varying vertical forcing which will be defined below.

b. The basic simulation and measured parameters of the flow in the vortex core

For purposes of comparison, we will return to the original F93 initial and boundary condi- 
tions for axisymmetric incompressible simulations in a closed domain. The domain lies in the range $0 \leq r \leq 2$ and $0 \leq z \leq 1$, with no-slip, solid-wall boundary conditions on all sides except on the center axis $r=0$. While F93 put his domain into solid-body rotation by incorporating a Coriolis term into the equations of motion, in our case this effect is reproduced by initializing the fluid in solid-body rotation at the same rate $\Omega=0.2$, and keeping the boundaries fixed at this rotation rate. The flow is driven by a fixed vertical forcing field in the form of a Gaussian bubble in the center of the domain:

$$
F_{z}(r, z)=1.264 e^{-20\left[r^{2}+(z-0.5)^{2}\right]} .
$$

In subsequent sections we will vary the location and shape of the convective forcing, so it is useful to rewrite (3.5) as

$$
F_{z}=C_{b} e^{-\left\{\frac{r^{2}}{\sigma_{h}{ }^{2}}+\frac{\left(z-z_{\text {forc }}\right)^{2}}{\sigma_{v}{ }^{2}}\right\}}
$$

where $C_{b}=1.264, z_{\text {forc }}=0.5$, and $\sigma_{h}=\sigma_{v}=0.2236$ are the horizontal and vertical length scales, respectively.

At $t=0$, the flow is at rest except for the solid-body rotation, i.e., $u=w=0$ and $v=\Omega r$. The development of a tornado-like vortex from fluid in solid-body rotation has been discussed extensively by Fiedler $(1993,1994)$ and NF99, so we will not provide a discussion of the development of the vortex, but instead address how well the AMR model reproduces the results with full resolution. For this purpose we ran three simulations of the F93 type: one with a fixed resolution of 256x128 gridpoints in the horizontal and vertical directions, respectively; and two with a base resolution of $64 \times 32$ gridpoints and a maximum of two levels of factor two refinement, so that the highest resolution in the regions of dynamical interest matched that of the fixed 
256x128 case. The two AMR simulations of the F93 simulation were different only in regards to their refinement conditions: one used as a refinement condition that $R e_{c e l l}$ [defined above in (2.1)] be less than 20 everywhere, whereas the other required $R e_{c e l l}<10$.

Fig. 3 shows the maximum vertical, azimuthal, and negative radial velocities in the three F93 simulations from $t=0$ to $t=70$. It is readily apparent that both AMR simulations do a fairly good job of reproducing the full grid results. One can also see that the $R e_{c e l l}<10$ simulation is consistently closer to the full grid results than the $R e_{c e l l}<20$ simulation. This is demonstrated even more clearly in Fig. 4, which shows close-ups of the maximum vertical and azimuthal velocities separately. While the $R e_{\text {cell }}<20$ simulation occasionally shows significant departures from the full grid solutions, the $R e_{c e l l}<10$ simulation follows the full grid solution very closely with occasional deviations on the order of $5 \%$ for the vertical velocities and even smaller for the azimuthal and radial velocities.

The AMR code with a properly chosen refinement condition (usually $R e_{c e l l}<10$ or less) quite accurately reproduces the results of a full grid simulation for short times. However, over the course of studying a wide variety of refinement conditions for long-time simulations we have found that the AMR code cannot reproduce the exact details of a full grid simulation for long times. As an example, consider the results shown in Fig. 5, which compares the azimuthal wind fields and the wind vectors in the $r-z$ plane (often called the meridional velocity vectors) at $t=100$ for the AMR simulation and the full-resolution simulation. For clarity, these vectors are generated using only the base-grid data in the AMR simulation, and are interpolated onto a $63 \times 32$ grid in the full-resolution simulation. The results are nearly indistinguishable for the azimuthal velocities in the vortex core. In the far field, where the resolution of the AMR simulation is lower than that of the full-resolution simulation, one can clearly see some differences. Fig. 6 shows the long-term 
evolution for each simulation in terms of the maximum windpseeds and the vortex aspect ratio. From these figures we can draw two conclusions: 1) after a period of nearly steady flow, the flow in the vortex core becomes unsteady with large oscillations in the vertical windspeeds and the vortex structure; 2) while the AMR simulation does not follow the evolution of the full resolution simulation exactly, it reproduces the behavior in a statistical sense. Mean values for $C_{\vartheta}$ the height of the maximum azimuthal winds (ZMW), the vortex aspect ratio $A_{V}$, and the standard deviation of $A_{V}$, are shown in Table 2 for the full resolution and the AMR simulation with $R e_{\text {cell }}<10$. Since it is generally observed that ZMW is coincident with the height where the radial inflow goes to zero, we consider it to be equivalent to the depth of the swirling boundary layer. These statistics were computed from $t=100$ to $t=200$ in the simulations. The mean $C_{v}$ are within $1 \%$ of each other for the two cases and the mean $A_{V}$ are within $2 \%$ of each other. The standard deviation of $A_{V}$ is $12 \%$ less in the AMR simulation. The reason for this difference is not clear, although one can speculate that the larger numerical dissipation inherent to the regions of coarser gridding could decrease the variance in the AMR case. Nonetheless, these results are very encouraging because we are not interested in the exact evolution of axisymmetric tornado-like vortices, but rather in the long-time average maximum velocities and vortex structures generated by a particular set of model parameters.

The utility of adaptive mesh refinement is illustrated by considering the differences in memory use and CPU time between the full resolution and AMR cases. The full resolution simulation used 256x128 = 32,768 grid cells, and took 754 seconds of CPU time on a Sun Ultra 1 processor to integrate the equations of motion from $t=200$ to $t=201$. The AMR simulation with $R e_{\text {cell }}$ $<10$ at $t=200$ had a base grid of $64 \times 32=2048$ grid cells, one level 1 grid covering $56.25 \%$ of the domain with 4608 cells, and three level 2 grids covering $10.74 \%$ of the domain with 3520 cells, 
for a total of 10,176 cells. The CPU time on the same processor from $t=200$ to $t=201$ was 274 seconds.

\section{Changes in Model Geometry}

In this section we explore how changes in the size of the domain and the location and shape of the convective forcing field affect the structure and maximum windspeeds of the vortex. The names for each simulation and their relevant parameters are summarized in Table 1. The resulting velocity coefficients, vortex aspect ratios, and boundary layer depths for all the simulations are summarized in Table 2. Except where noted, these statistics were computed from $t=100$ to $t=200$ in each simulation.

\section{a. Simulations with larger domains}

Using a closed domain with a ceiling at $z=1$ certainly seems restrictive when using a convective forcing field that is maximized at $z=0.5$ and when the outflow from the vortex core clearly impinges on the ceiling, especially during the initial evolution of the vortex (see F93 and NF99). Fiedler $(1993,1994)$ argued that the ceiling at the top of the domain was analogous to the tropopause, and the vertical extent of the domain was meant to represent the entire troposphere. While this may be valid for the main updrafts in supercell thunderstorms, not all atmospheric tornadolike vortices are associated with convection that reaches the tropopause (i.e., waterspouts, dust devils). Thus it is worthwhile to determine the effect of moving the "ceiling" further away from the surface.

Our first step was to repeat the F93 simulation but with the upper boundary of the domain moved up to $z=2$, using AMR with a base grid of $64 \times 64$ gridpoints and an unlimited number of levels of refinement. This is simulation F93ZDBL. The time evolution of the maximum velocities and the vortex aspect ratio are shown in Fig. 7 and the azimuthal wind field and meridional veloc- 
ity vectors are shown in Fig. 8. The vortex which ultimately develops in this simulation is essentially identical to the one which develops in the original F93 simulation, especially near the surface. The notable difference in the results is that when the domain height is increased the vortex transitions much more rapidly to the unsteady dynamics observed in the last 100 time units of the F93 simulation, and it appears that the vortex is a bit more unsteady than before. Also, the vortex outflow, as indicated by the meridional velocity vectors in Fig. 8b, extends higher and further out than in the case with the ceiling at $z=1$.

Another important observation is that the downward flow in the core of the vortex appears to be at least as vigorous as in the F93 simulation. This suggests that in the previous case the proximity of the ceiling did not enhance this downward flow. Additional simulations with still higher upper boundaries, and also outer boundaries that were further from the axis (not shown), gave nearly identical results.

\section{b. Convective forcing further from the surface}

The way in which the evolution of the vortex depends on the altitude and the shape of the convective forcing field has previously been investigated by Trapp and Davies-Jones (1997). Using the same axisymmetric model as Fiedler (1994), with similar boundary conditions and convective forcing functions, they investigated under what circumstances the tornado-like vortex formed by a mechanism known as the dynamic pipe effect (DPE). A DPE occurs when the lowest levels of the convective forcing are sufficiently far above the surface. At first, the convective forcing induces convergence of the rotating fluid only immediately below. The amount of actual fluid convergence which occurs is limited by local increase in the centripetal force, but the local intensification of the rotation creates a region of lower pressure beneath the convective forcing. This low pressure induces more convergence below, which then induces more low pressure and so on. 
By this mechanism the effect of convective forcing at high altitudes is propagated downwards until it reaches the surface, where due to interaction with the surface an intense vortex forms.

In this paper we focus not on the initial development of the vortex but on the long-term windspeeds and structure of the vortex which forms. Simulation HIBUBL was the same as F93ZDBL except that the height of the convective forcing was changed to $z_{\text {forc }}=1.0$. The results are nearly identical to what we have seen before, except that the vortex is less unsteady and with a slightly lower aspect ratio. While the flow field near the surface is shown in Fig. 9 to be essentially the same as what we have seen before, the tornado outflow does not diverge away from the center axis at an altitude of $z=0.4$, but instead continues upwards and even appears to re-accelerate as it approaches the convective forcing. We also see secondary maxima in the azimuthal and vertical velocities in the vicinity of $z=0.9, r=0.2$. These secondary maxima are caused by a local intensification of the rotation and vertical motion caused by the convective forcing field. Additional simulations with convective forcing at still higher altitudes (not shown) gave similar results.

\section{c. The shape of the convective forcing}

In the analysis of NF99, it was assumed that there was only one important length scale in the determination of the character of the vortex. This length scale was assumed to be the vertical height of the domain, which also happened to be equal to the vertical and horizontal scales of the convective forcing. However, we have already shown that the size of the domain and the height of the convective forcing have little impact on the character of the vortex.

It remains to be seen how the vertical and horizontal dimensions of the convective forcing affect the results. For these simulations, the convective forcing remains centered at the altitude $z_{\text {forc }}=1.0$. We proceed by first doubling the vertical extent of the convective forcing in a simulation called TALLBUBL. However, since the convective velocity scale $U$ is determined by the inte- 
gral of the forcing along the vertical axis, to maintain $U=1$ we decrease the magnitude of the forcing by a factor of one half, i.e, $C_{b}=0.632$. The results of such a simulation are summarized in Table 2 and we can see that there has been no appreciable change in the velocity coefficient or the aspect ratio.

Next, we return the vertical extent of the forcing to its original size and then double its horizontal extent for simulation WIDBUBL. Theoretically, this should not cause a change in the velocity scale. The resulting mean velocity coefficient of $C_{v}=0.7601$ is slightly higher, but clearly more significant are the changes in the mean and variance of $A_{V}$ which are 3.8854 and 0.7808 , respectively. Increasing the horizontal extent of the convective forcing has substantially changed the structure of the vortex. The change can be seen in a snapshot of the azimuthal wind fields and the meridional velocity vectors, shown in Fig. 10, where it is apparent that the vortex has a twocelled structure with a very wide core. What is the reason for this change? NF99 claimed that the structure should only depend on $R e_{V}$ - but is in fact the structure also closely tied to the horizontal scale of the convective forcing?

Since the fluid in the far-field is in solid-body rotation, the circulation of the fluid increases with the square of the distance from the axis. If the convective circulation reaches out farther from the axis, the fluid it brings into the tornadic vortex will have a substantially higher circulation. Since the circulation of the fluid drawn into the vortex scales as $\Gamma \sim \Omega L^{2}$, we should be able to make a vortex with the same $R e_{V}$ by reducing $\Omega$ to $25 \%$ of its previous value. To test this hypothesis, we ran simulation WIDBUBL $\Omega .05$ with the same, wider convective forcing but with one quarter the rotation rate, $\Omega=0.05$. The results, shown in Fig. 11 and Table 2, indicate that the vortex structure is once again that of a DVJ with an mean $A_{V}=1.4085$. It also appears that wider convective forcing allows for slightly higher windspeeds as compared to the F93 and HIBUBL 
simulations. Further simulations with different convective forcing widths and rotation rates confirmed these observations. In regards to predicting the structure of the vortex, the appropriate length scale to use in the calculation of $R e_{V}=\Gamma / v=\Omega L^{2} / \mathrm{v}$ is in fact the width of the convective forcing. This, however, only applies to simulations with solid-body rotation in the far-field.

Another interesting result is that the depth of the swirling boundary layer (ZMW) and the radius of maximum winds (RMW) are both approximately twice as large in simulation WIDBUBL $\Omega$.05. The change in the depth of the boundary layer is surprising, since it did not increase in depth when the only change was to make the convective forcing wider. The relationship between the length scales in the vortex core and the horizontal scale of the convective forcing will be explored further in section 7 .

\section{Results for Higher Reynolds Numbers}

Some of the claims made by NF99 were in regards to how the vortex behaves as the eddy viscosity $v$ is decreased. In particular, NF99 claimed that 1) as the viscosity is decreased, the circulation of the fluid must be decreased proportionally in order to keep the same vortex structure; 2) if one does decrease the circulation accordingly, the mean maximum windspeeds would increase; and 3) the maximum windspeeds were observed when the vortex was in a "low-swirl," one-celled vortex structure. In the following sections we evaluate these claims using the AMR model.

\section{a. Vortex structure}

The obvious first step in investigating how the vortex structure changes at higher Reynolds numbers is to increase the amplitude of the convective forcing. In simulation F93DBLU, the convective forcing was increased by a factor of $4\left(C_{b}=5.07\right)$ so that the convective velocity scale was doubled, i.e., $U=2.0$. After a short time, the simulation produced a tornado-like vortex that was 
nearly identical to those seen before, except that the velocities throughout the vortex core were almost exactly doubled, as indicated by the results in Table 2 . This confirms that increasing the strength of the convective forcing does not have the same effect as increasing the flow rate through a tornado vortex chamber, and that the vortex Reynolds number $\Gamma / \nu$ is the important parameter for predicting vortex structure.

By substantially increasing the background rotation rate $\Omega$, one can easily cause the structure of the vortex to change from a drowned vortex jump to that of a two-celled vortex. We call this simulation HISWIRL2CELL. An snapshot of the flow in this simulation is shown in Fig. $12 \mathrm{ab}$, which is the result of an F93-type simulation with $\nu=0.0005$ and $\Omega=0.5$. The vortex Reynolds number $R e_{V}=1000$ produces a vortex that jumps back and forth between two-celled and drowned vortex jump structures but spends most of its time in the two-celled state. These transitions are caused when large-amplitude axisymmetric rolls, which are propagating down the vortex core, reach the surface (NF99, sec. 6a). The vortex statistics are shown in Table 2.

Next we consider a similar simulation with $R e_{V}=1000$, but instead with $v=0.0002$ and $\Omega=0.2$, which is referred to as LOWSWIRL2CELL. This results again in a vortex with a predominantly two-celled structure, as shown in Fig. 12cd. The instantaneous flow structure is clearly more complex in this case due to the substantially lower viscosity, but the overall structure of the vortex is quite similar to that in HISWIRL2CELL. The lower viscosity also allows for a higher mean windspeed with $C_{v}=0.8104$.

\section{b. Maximum windspeeds}

By decreasing $\Omega$ accordingly, we can recover the drowned vortex jump structure for the vortex when the model has lower viscosities, as in the previous section. The simulation LOWSWIRLDVJ refers to the case where $\Omega=0.08$ and $v=0.0002$. The drowned vortex jump 
structure is recovered (not shown) and we find $C_{v}=0.7972$. While this is slightly higher than in all the F93-type simulations, it is smaller than that found above with the same viscosity and $\Omega=0.2$. In contrast to what was found by NF99, decreasing the rotation rate $\Omega$ so that the vortex evolved towards a "one-celled" structure does not in fact increase the maximum windspeeds. This was confirmed by the results of additional simulations with still smaller values for $\Omega$ (not shown). Rather, we find that the highest windspeeds are found with higher circulations that produce widebased, two-celled vortices. Possible reasons for this difference will be discussed in the Conclusions.

We have endeavored to find the highest mean azimuthal windspeeds that our closeddomain model can produce. We have previously found that the windspeeds increase for smaller viscosities, and that the two-celled vortex structures have the highest windspeeds. We also saw above that the maximum windspeeds were somewhat higher for wider convective forcing fields. Despite the computational advantages offered by adaptive mesh refinement, the computational cost for equal amounts of simulation time increases drastically with decreasing eddy viscosity $v$. With these points in mind, we ran simulation LOWESTVISC which had $\Omega=0.1, v=0.0001$, and the wider convective forcing field with $\sigma_{h}=0.4472$. The resulting tornado-like vortex had substantially higher mean windspeeds than any of the previous simulations with a mean $C_{v}=1.04$. The vortex core was very wide, with $A_{V}=4.06$, and flow in the core was very unsteady, as summarized by the results in Table 2 .

\section{Tornado-Scale Simulations}

\section{a. Motivation}

Let us summarize the important conclusions drawn from the solid-body rotation simulations: 
1. Increasing the size of the domain has little effect on the vortex structure and maximum windspeeds.

2. The altitude (height) of the convective forcing has little effect on low-level vortex structure and maximum windspeeds. The vertical extent (depth) of the convective forcing also does not affect the vortex structure, and determines the low-level windspeeds only to the extent to which it contributes to the convective velocity scale (the effective CAPE).

3. The width (horizontal scale) of the convective forcing determines the vortex structure to the extent to which it determines the circulation of the fluid that is drawn into the vortex core. Increasing the width also produces somewhat higher windspeeds, and seems to affect the depth of the boundary layer in some cases.

4. Decreasing the viscosity allows for higher windspeeds and decreases the depth of the swirling boundary layer.

These conclusions were drawn entirely from simulations where the fluid in the far field was in solid-body rotation. A natural question to ask is whether or not the same conclusions apply when the circulation in the far field reaches a finite limit.

Fiedler (1993) commented that the tornado-like vortices produced in his model were crude models of actual tornadoes, because their internal length scales were an order of magnitude larger than those observed. Our results show these length scales are not related to the size of the domain (i.e., the height of the tropopause), but rather to the properties of the environmental forcing and the eddy viscosity. In the next section we will use these ideas to produce tornado-like vortices on dimensional scales which match the characteristic scales of observed tornadoes.

\section{b. Mesocyclone-scale domain and forcing}

We now construct a simple, axisymmetric model of the interaction of a thunderstorm-scale 
updraft with mesocyclone-scale vortex, using dimensional scales in accord with observations. We again use a closed domain and a fixed convective vertical forcing field, but we re-write the magnitude of the forcing in terms of a maximum temperature anomaly $T^{\prime}$ and a mean background temperature $\bar{T}$ in a Boussinesq fluid:

$$
F_{z}=g\left(\frac{T^{\prime}}{\bar{T}}\right) e^{-\left\{\frac{r^{2}}{\sigma_{h}{ }^{2}}+\frac{\left(z-z_{\text {forc }}\right)^{2}}{\sigma_{v}{ }^{2}}\right\}}
$$

where $g$ is the gravitational acceleration.

Rather than putting the system into solid body rotation, we instead construct a more realistic rotational environment. The azimuthal velocity field is initialized as a Rankine vortex with a maximum windspeed $V^{*}$ that occurs at some radius of maximum winds ${ }^{2} r_{\max }$. However, unlike the solid-body rotation simulations, the azimuthal velocity is set to zero at the surface, and the transition between the no-slip surface and the Rankine vortex aloft is modelled with a logarithmic profile. Explicitly:

$$
v_{0}(r, z)= \begin{cases}f(z) V^{*} r / r_{\max } & r \leq r_{\max } \\ f(z) V^{*} r_{\max } / r & r>r_{\max }\end{cases}
$$

with

$$
f(z)=\left\{\begin{array}{cc}
0 & z<z_{0} \\
\frac{\log \left(z / z_{0}\right)}{\log \left(z_{\text {top }} / z_{0}\right)} & z_{0} \leq z \leq z_{\text {top }} \\
1 & z>z_{\text {top }}
\end{array}\right.
$$

where $z_{0}$ is the top of the friction layer and $z_{\text {top }}$ is the top of the logarithmic layer. By setting $v$ to

2. Not to be confused with RMW, the radius of maximum wind of the tornado-like vortex. 
zero below $z_{0}$ we are neglecting the azimuthal wind in the friction layer; alternatively we could consider $z_{0}$ to be a roughness length below which there is no meaningful flow. The fluid outside of $r_{\max }$ represents an unlimited supply of fluid with a constant circulation $\Gamma=r_{\max } V^{*}$. To maintain this unlimited supply, we keep the circulation approximately constant in the far field (far from the tornado-like vortex) by adding a forcing term to the azimuthal momentum equation which continually drives the azimuthal velocity beyond $r_{\max }$ back to this constant value, i.e.,

$$
F_{\theta}(r, z)=\left\{\begin{array}{cc}
0 & r<r_{\text {max }} \\
-\tau^{-1}\left[v(r, z)-v_{0}(r, z)\right] & r \geq r_{\text {max }}
\end{array}\right.
$$

where $\tau$ is a time scale for the relaxation. The upper and lateral boundaries are changed to freeslip conditions, since they are no longer needed to maintain the supply of angular momentum.

In recent years, a number of field studies - particularly those associated with project VORTEX (Rasmussen et al., 1994) - have produced remarkably detailed observations of the interior environment of supercell thunderstorms (Wakimoto and Atkins, 1996; Bluestein et al., 1997, Wakimoto et al., 1998; Wakimoto and Liu, 1998; Trapp, 1999). Reasonable choices for length and velocity scales for tornado-scale simulations can be drawn from these observations. For the larger, mesocyclone-like vortex, we let $r_{\max }=2 \mathrm{~km}$ and $V^{*}=15 \mathrm{~ms}^{-1}$. The depth of the mesocyclone swirling boundary layer is chosen to be $z_{\text {top }}=300 \mathrm{~m}$ and the roughness length $z_{0}=1 \mathrm{~m}$. The far-field circulation is relaxed back to its original value at a time scale $\tau=20 \mathrm{~s}$.

We use a closed domain with an upper boundary at $z=8 \mathrm{~km}$ and an outer boundary at $r=8 \mathrm{~km}$. While $8 \mathrm{~km}$ is considerably lower than the mid-latitude tropopause, it is approximately equal to the scale height of the atmosphere, which is also equal to the depth the atmosphere would take if the same mass of fluid were incompressible with constant density, as is the 
case in our model. The convective forcing field is centered at $z_{f o r c}=4 \mathrm{~km}$, with vertical and horizontal scales $\sigma_{v}=\sigma_{h}=2 \mathrm{~km}$. The maximum temperature anomaly is $T^{\prime}=20 \mathrm{~K}$ in an environment with a mean temperature $\bar{T}=280 \mathrm{~K}$. The initial azimuthal velocity and the convective forcing field (in terms of temperature anomaly) are shown in Fig. 13. The convective forcing has $2469 \mathrm{~J} \mathrm{~kg}^{-1}$ of CAPE with an associated convective velocity scale $U=70.3 \mathrm{~ms}^{-1}$. While the maximum temperature anomaly is quite a bit higher than the maximum temperature anomalies usually predicted by highly unstable environmental soundings, this accounts for the contracted vertical scale of the convection and allows for a CAPE typical of strong thunderstorms. The initial base grid has 256 points in the radial and vertical directions, such that $\Delta r=\Delta z=31.25 \mathrm{~m}$. We set $v=40 \mathrm{~m}^{2} \mathrm{~s}^{-1}$.

Snapshots of the circulation $\Gamma$ and vertical velocity fields at $t=1616 \mathrm{~s}$ are shown in Fig. 14. These results show a scale separation between the full model domain, the convective forcing, and intense, low-level vortex; note how small the tornado-like vortex is in comparison with the largescale features of the flow and the rest of the domain. A close-up of the azimuthal velocity field of the near-surface vortex is shown in Fig. 15, which in this case exhibits a drowned vortex jump structure nearly identical in shape to many of the vortices generated in the solid-body rotation experiments. The RMW at this time is $158 \mathrm{~m}$ and the ZMW is $119 \mathrm{~m}$, consistent with the observations of Wurman et al. (1996). The velocities are also comparable, with $V_{\max }=73 \mathrm{~ms}^{-1}$ versus the $70 \mathrm{~ms}^{-1}$ observed. Thus we find that a tornado-like vortex very similar in structure to the one observed by Wurman et al. (1996) can be produced with a far-field circulation associated with a maximum azimuthal velocity of $15 \mathrm{~ms}^{-1}$ at $2 \mathrm{~km}$ radius, and a constant eddy viscosity of $40 \mathrm{~m}^{2} \mathrm{~s}^{-}$

1. The vortex reaches a quasi-steady state roughly after $t=1500 \mathrm{~s}$, with mean values for $C_{v}=0.83$ $\left(\right.$ mean $\left.V_{\max }=64.3 \mathrm{~ms}^{-1}\right), A_{V}=1.66, \mathrm{RMW}=188.2 \mathrm{~m}$, and $\mathrm{ZMW}=116.2 \mathrm{~m}$. 
Examination of the secondary (meridional) circulation in the tornado-scale simulations reveals some features which are quite different from the solid-body rotation simulations. Fig. 16 shows a vector plot of the meridional flow, interpolated onto a $32 \times 32$ grid, at the same time as the instantaneous velocity fields shown in Fig. 14 and Fig. 15. On the larger scale, the meridional circulation is very different than what was seen in the solid-body rotation simulations. In the solidbody rotation simulations, the radial inflow of the swirling boundary layer begins substantially farther from the axis than the width of the convective forcing, and continues all the way into the core of the vortex (see, e.g., Fig. 5). In the tornado-scale simulation, however, one can clearly identify two distinct radial inflow regions. The outer region begins at the domain's outer boundary and flows inward to about $r=4 \mathrm{~km}$, then separates from the surface and flows up through the convecting region. Interestingly, this mesocyclone-scale meridional circulation is quite similar to that analyzed by Trapp (1999, Fig.7) from radar observations of a nontornadic supercell. The second radial inflow region is much smaller in scale and is clearly associated with the tornado-like vortex, as shown in Fig. 15b; however, the radial extent of the smaller radial inflow region is substantially less than the width of the convective forcing. As a result of the separation of the outer boundary layer, the circulation of the fluid which does arrive in the tornado core is substantially lower than the circulation in the far field, as can be seen from inspection of Fig. 14b. Indeed, the vortex Reynolds number that would be associated with the circulation in the far field $\left(3.0 \times 10^{4} \mathrm{~m}^{2} \mathrm{~s}^{-1} / 40 \mathrm{~m}^{2} \mathrm{~s}^{-1}=750\right)$ is much higher than the values typically associated with a drowned vortex jump. The large radial gradients of $\Gamma$ which can be seen in Fig. 14b near the center axis suggests that the flow has an azimuthal velocity profile much closer to solid-body rotation than to potential flow. The tornado-like vortex, and its more intense swirling boundary layer, appears to form within this region and its internal length scales may possibly be determined by the 
local rotation rate of the overhead fluid, rather than specifically by the circulation in the far-field.

One must also consider that this double-structure of the meridional circulation may be related to the dimensional scales of these simulations, or to the fact that the mesocyclone vortex is required to transition to zero flow at the surface via the log-layer described in (6.3). To address this possibility, we performed some additional F93-type simulations where the circulation in the far-field was held at a constant value, the log-layer was removed, and the lower boundary was also required to rotate with the fluid aloft. The results of these simulations were strongly dependent on the choice for the radius $r_{\max }$ of the solid body rotation core, and some examples are shown in Fig. 17. In each case, $V^{*}$ was chosen so that the core rotation rate remained $\Omega=0.2$. With $r_{\max }=1.0$, the results are nearly identical to those simulation F93AMR. However, with $r_{\max }=0.5$, a near-surface vortex fails to form, even though the solid-body core is 2.5 times wider than the convective forcing. These results indicate that the successful development of a tornado-like vortex is strongly dependent on the existence of a sufficiently broad core of vorticty in the rotating environment.

The double-structure of the meridional circulation casts doubt as to whether similar dependencies on the environmental parameters found in the solid-body rotation simulations will apply to simulations with finite circulation in the far-field (see section 6a). We performed three additional tornado-scale simulations. The parameters for all four tornado-scale simulations are outlined in Table 3, and the statistical results of long simulations (averaged from $t=1800 \mathrm{~s}$ to $t=2400 \mathrm{~s}$ ) are shown in Table 4 . The changes in vortex structure are qualitatively consistent with the changes expected from the results of the solid-body rotation simulations: simulation TORNADOHIVORT, with twice the circulation in the far-field, has a substantially wider core; TORNADOHIVISC, with twice the eddy viscosity, has a deeper boundary layer and a lower aspect ratio 
( $A_{V} \approx 1.0$ ), and TORNADOWIDE, with $50 \%$ wider convective forcing, has a slightly deeper boundary layer. Other aspects, however, are not consistent; for example, the mean velocity coefficient $C_{v}$ is very low for the simulation with the wider convective forcing.

\section{Length Scales in Tornado-Like Vortices}

Analytical and numerical methods have been applied to the study of swirling boundary layers for some time. In the case where the fluid above the surface is in solid body rotation, a classical Ekman layer develops, as shown by Greenspan and Howard (1963) [see also the text by Greenspan (1990)]. Eliassen (1971) and Eliassen and Lystad (1977) extended these results to the cases with modified lower boundary conditions (to account for turbulence) beneath vortices with varying velocity profiles. They found that a similar boundary layer develops, and that the depth of the boundary layer can be predicted from

$$
\delta=\left(\frac{2 v}{N}\right)^{\frac{1}{2}}
$$

where

$$
N=r^{-3} \frac{\partial}{\partial r}\left(\Gamma(r)^{2}\right)
$$

is the inertial stability of the overhead vortex.

Unfortunately (7.1)-(7.2) fails for a potential (1/r) vortex. The early work of Barcilon (1967) and Carrier (1971) on boundary layers beneath potential vortices identified the importance of the vortex Reynolds number $\Gamma / v$. Burggraf et al. (1971) numerically integrated the equations of motion beneath a potential vortex and found that the depth of the boundary layer scales as

$$
\delta=\left(\frac{v}{\Gamma}\right)^{\frac{1}{2}} r_{b}
$$


where $r_{b}$ indicates the distance from the center axis where the boundary layer begins (as in the case with a finite disc of radius $r_{b}$ immersed in rotating fluid). With tornadoes specifically in mind, Kuo (1971) attempted a similar calculation under the assumption that the overhead potential vortex was maintained in intensity. He did find some properties similar to those of observed and simulated tornadoes, such that there is a local maximum in the azimuthal velocity generated near the surface, and that the boundary layer depth increases towards the center axis.

We have observed that the depth of the boundary layer (ZMW) and the radius of maximum winds (RMW) show some dependence on the horizontal scale of the convective forcing. Furthermore, in solid-body rotation simulations, this horizontal scale determines the circulation of the fluid that is drawn into the vortex core. It therefore appears that the horizontal scale of the convective forcing, which we shall label $L_{h}$, is the best candidate for the fundamental length scale in the problem [i.e., it should be used for $L$ in (1.1) and (1.3)]. We also have the convective velocity scale $U$, the far-field circulation $\Gamma$, and the eddy viscosity $v$. NF99 showed that four different dimensionless parameters can be constructed from these four dimensional scales, and that any two (but only two) of them can be used together to describe the full range of possible outcomes. NF99 also found that the most useful choices of these four parameters to use together were the vortex Reynolds number $R e_{V}$ and the convective Reynolds number $R e_{C}$, by showing that 1) the vortex structure depended almost exclusively on $R e_{V}$, and 2) for fixed $R e_{V}$, the maximum windspeeds increased with increasing $\operatorname{Re}_{C}$. The small increase in maximum windspeeds seen with wider convective forcing also supports using $L_{h}$ in the definition of $R e_{C}$.

For the length scales in the vortex core, such as the depth of the swirling boundary layer, dimensional analysis suggests the following relationship: 


$$
\mathrm{ZMW}=C_{\mathrm{ZMW}} f\left(R e_{C}, R e_{V}\right) L_{h}=C_{\mathrm{ZMW}} f\left(\frac{U L_{h}}{\mathrm{v}}, \frac{\Gamma}{\mathrm{v}}\right) L_{h}
$$

where $C_{\mathrm{ZMW}}$ is an unknown coefficient. Through a careful examination of our results, we can try to guess the nature of the function $f$. First, the results indicate that $f$ is an only very weakly varying function of $R e_{C}$, possibly even constant for large values. This can be seen by comparing the results of simulations HISWIRL2CELL and LOWSWIRL2CELL. Between these two simulations, $R e_{V}$ was held constant, but $v$ was decreased by $60 \%$, requiring a large change in $R e_{C}$. Yet, the mean value of ZMW decreased only $4.5 \%$. This suggests that to a first approximation we may neglect the variation of $\operatorname{Re}_{C}$ in $f$.

In simulation WIDBUBL, we doubled $\sigma_{h}$, and yet the mean ZMW decreased slightly, rather than doubling in size. To resolve this discrepancy, recall that in the solid-body rotation simulations, the circulation scales as $\Gamma \sim \Omega \sigma_{h}^{2}$ (here we assume $L_{h} \propto \sigma_{h}$ ). Thus, changes in $L_{h}$ alone will not cause a change in ZMW provided that the function $f\left(R e_{V}\right)$ has the form $f\left(R e_{V}\right)=R e_{V}^{-1 / 2}$. (The slight decrease in ZMW may be accounted for by a slight decrease in $f$ with increasing $R e_{C} \propto L_{h}$, which we are neglecting.) This suggests the following simplified relationship for the depth of the boundary layer:

$$
\mathrm{ZMW}=C_{\mathrm{ZMW}}\left(\frac{\mathrm{v}}{\Gamma}\right)^{\frac{1}{2}} L_{h}
$$

It is interesting to note that in the solid-body rotation case, $(7.5)$ predicts $\mathrm{ZMW} \sim(\mathrm{V} / \Omega)^{1 / 2}$, which is identical to the depth of the classic Ekman boundary layer (Greenspan, 1990, p. 36), whereas if the circulation in the far-field is constant, we have recovered (7.3) - the results of Burggraf et al., (1971). If this relationship were accurate, then a single value of $C_{\mathrm{ZMW}}$ should be consistent with the results from all the simulations. Based on the observed mean values of ZMW, we 
computed the corresponding values of $C_{\mathrm{ZMW}}$ in all solid-body rotation simulations. The results are summarized in Table 2 . The computed values lie in the range $1.32<C_{\mathrm{ZMW}}<1.51$, with a mean of 1.42 .

Can a similar analysis be used to predict RMW? If a parcel of fluid did not lose any angular momentum as it was advected towards the center axis, we would expect it to achieve the maximum velocity (equal to the CAPE velocity scale if $C_{v} \sim 1$ ) at $r=\Gamma / U$. dimensional analysis then suggests

$$
\mathrm{RMW}=C_{\mathrm{RMW}}\left(\frac{\Gamma}{U}\right) g\left(R e_{V}, R e_{C}\right)
$$

Again, we will use our results to attempt to infer the properties of the function g. NF99 (see also Fig. 2) found that the vortex aspect ratio was essentially a function of $\operatorname{Re}_{V}$ and had only a weak dependence on $\operatorname{Re}_{C}$. To a first approximation, then,

$$
A_{V}=\frac{\mathrm{RMW}}{\mathrm{ZMW}}=\frac{C_{\mathrm{RMW}}(\Gamma / U) g\left(R e_{V}, R e_{C}\right)}{C_{\mathrm{ZMW}}(v / \Gamma)^{1 / 2} L_{h}}
$$

should in fact be approximately a function of $R e_{V}$ only. To make this so, let us first assume that $g$ is separable, i.e., $g\left(\operatorname{Re}_{V}, \operatorname{Re}_{C}\right)=h\left(\operatorname{Re}_{V}\right) k\left(\operatorname{Re}_{C}\right)$. Now, with some manipulation, (7.7) can be rewritten as

$$
A_{V}=C_{A}\left(R e_{V}\right)^{\frac{3}{2}} h\left(R e_{V}\right) k\left(R e_{C}\right)\left(R e_{C}\right)^{-1} .
$$

For $A_{V}$ to be (approximately) only dependent on $R e_{V}$, then we must have $k\left(R e_{C}\right) \sim R e_{C}$. (7.6) then becomes

$$
\mathrm{RMW}=C_{\mathrm{RMW}}\left(\frac{\Gamma}{U}\right) h\left(\operatorname{Re}_{V}\right) R e_{C} .
$$


All that remains is to estimate the form of the function $h\left(R e_{V}\right)$. Between the HIBUBL simulations and the WIDBUBL simulations, the RMW approximately increased by a factor of 2.38 , while the only environmental change was a doubling in the width $\sigma_{h}$ of the convective forcing. Using $\Gamma=\Omega \sigma_{h}^{2}, R e_{V}=\left(\Omega \sigma_{h}^{2}\right) / v$, and $R e_{C}=\left(U \sigma_{h}\right) / v$, we find a doubling of $\sigma_{h}$ will cause a 2.38 increase in RMW if $h\left(R e_{V}\right)=R e_{V}^{-0.8743}$. Thus we arrive at an approximate scaling law for the radius of maximum winds

$$
\mathrm{RMW}=C_{\mathrm{RMW}}\left(\frac{\Gamma}{U}\right)\left(\frac{v}{\Gamma}\right)^{0.8743}\left(\frac{U L_{h}}{\mathrm{v}}\right)
$$

Again, to test the validity of this scaling law, we use the steady-state results of all the simulations to solve for $C_{\text {RMW }}$; if $C_{\text {RMW }}$ is the same for all the simulations, the scaling law is valid. The results of these calculations are shown in Table 2, where it is shown that $0.182 \leq C_{\mathrm{RMW}} \leq 0.3829$ with a mean value of 0.3366 . The value of 0.182 is a significant outlier that which results from the LOWESTVISC simulation. This anomalous value may be a result of neglecting the effect of the convective Reynolds number $\operatorname{Re}_{C}$ on the vortex structure, since $R e_{C}$ is at least 4 times higher in this simulation than all the others.

Finally, we must ask whether or not these scaling laws also apply to the case where the circulation in the far-field is finite, rather than in solid body rotation. Using the scaling laws (7.5) and (7.10), we computed values for $C_{\mathrm{ZMW}}$ and $C_{\mathrm{RMW}}$ for the tornado-scale simulations. The results are shown in Table 4 . We find that the values for $C_{\mathrm{ZMW}}$ are somewhat consistent between the solid-body rotation simulations and the tornado-scale simulations. The values of $C_{\mathrm{RMW}}$, while being near in value to each other among the tornado-scale simulations, are all an order of magnitude smaller than in the solid-body rotation simulations. This inconsistency is caused by the ambi- 
guities in the circulation and horizontal length scales that are caused by the double-structure of the meridional circulation in the tornado-scale simulations.

\section{Conclusions}

We have used adaptive mesh refinement to clarify and expand upon the previous results of Nolan and Farrell (1999a). The important conclusions are summarized in the beginning of section 6 above. The results indicate that the structure and maximum windspeeds of tornadoes are less dependent on the "details" of the storm environment than one might expect. In particular, the maximum windspeeds of tornadoes are almost solely dependent on the vertically integrated intensity of the overhead convection, with only a relatively weak dependence on the structure of the vortex (as indicated by the fact that the velocity coefficient lies in the relatively narrow range $\left.0.7<C_{v}<1.0\right)$. Furthermore, this intensity has only a weak dependence on the shape of the convection which sustains it. We do find that the highest windspeeds occur when the vortex has a two-celled structure with a wide base, which is in contrast with the earlier findings of NF99.

The reason for the difference between our results and those of NF99 likely has to do with numerical resolution in the boundary layer. As the vortex transitions to a two-celled state, the swirling boundary layer becomes progressively shallower. Under-resolution of this layer will lead to spurious dissipation of radial and angular momentum, ultimately causing lower mean windspeeds. While it has generally been reported in the literature that the highest windspeeds occur when the vortex is in the DVJ state, some laboratory studies have found higher windspeeds in the high-swirl regime (Baker and Church, 1979). Fiedler (1994, 1998) also found the highest mean azimuthal windspeeds occurring in two-celled vortices. Certainly, this would be consistent with the general observation that the most damaging tornadoes usually have wide bases.

Our results did agree with the findings of NF99 that the structure of the vortex depends on 
the ratio of the circulation of the fluid that is drawn into the vortex to the eddy viscosity of the fluid, i.e., the vortex Reynolds number $\Gamma / v$. Furthermore, by using dimensional scales for the convection and far-field circulation that are consistent with the tornado environment, we found that realistic values for the maximum windspeeds, the radius of maximum winds, and the depth of the tornadic boundary layer can be reproduced using typical values for the eddy viscosity such as $v=40 \mathrm{~m}^{2} \mathrm{~s}^{-1}$; however, a minimum grid spacing of 3.9 meters (in an $8 \mathrm{~km} \mathrm{x} 8 \mathrm{~km}$ domain) was necessary to sufficiently resolve the dynamics. Since the effect of diffusion is by far the greatest in the near-surface boundary layer, the physical significance of the eddy viscosity carries over to the turbulence generated by surface roughness in actual tornadoes. This suggests that identical thunderstorm environments may produce very different tornadoes over different surfaces, i.e., land versus water, city buildings versus open plains. This is also consistent with observations and laboratory experiments [see, for example, the review by Church and Snow (1993) and the references therein.]

Certainly, asymmetric and three-dimensional processes are prevalent in tornadoes and will likely be critical in developing a complete understanding of these and other intense atmospheric vortices. Fiedler (1998) has shown that substantially higher transient, localized windspeed maxima are observed in three-dimensional models of tornado-like vortices. These instantaneous, highest windspeeds are associated with the smaller scale "suction vortices" that form in the region of large shear between the RMW and the relatively stagnant core. Furthermore, both numerical simulations (Lewellen et al., 1997) and recent theoretical work on asymmetric vortex dynamics (Nolan and Farrell, 1999b) indicate that three-dimensional dynamics enhance the mean, azimuthally averaged windspeeds as well as the instantaneous maxima. Fortunately, continuing advances in computer speed and memory will allow for more three-dimensional modelling at higher and 
higher resolutions, along with higher-order turbulence closure schemes. It is also encouraging to note that the speed and memory savings associated with adaptive mesh refinement in two dimensions will be even more substantial in three dimensions. 


\section{Acknowledgements}

The authors would like to thank Profs. A. J. Chorin and G. I. Barenblatt for helpful discussions on dimensional analysis and fluid dynamics, and Dr. V. Larson for helpful comments on an earlier version of the manuscript. D. Nolan would also like to thank Prof. M. T. Montgomery for many helpful discussions and references to earlier work. This work was supported in part by the Office of Science, Office of Advanced Computing Research, Mathematical, Information, and Computational Sciences Division, Applied Mathematical Sciences Subprogram, of the U. S. Department of Energy, under Contract No. DE-AC03-76SF00098. D. Nolan was also supported by the Office of Naval Research under contract N00014-93-1-0456 P0006, and by Colorado State University. 


\section{References}

Almgren, A. S., J. B. Bell, P. Colella, L. H. Howell, and M. L. Welcome, 1998: A conservative adaptive projection method for the variable density incompressible Navier-Stokes equations. J. Comp. Phys., 142, 1-46.

Almgren, A. S., J. B. Bell, and W. Y. Crutchfield, 1999: Approximate projection methods: Part I. Inviscid analysis. Submitted to SIAM J. Sci. Comput. Also available from the Lawrence Berkeley National Laboratory as report LBNL-43374.

Almgren, A. S., J. B. Bell, and W. G. Szymczak, 1996: A numerical method for the incompresible Navier-Stokes equations based on an apporximate projection. SIAM J. Sci Comput., 17.

Baker, G. L., and C. R. Church, 1979: Measurements of core radii and peak velocities in modelled atmospheric vortices. J. Atmos. Sci., 36, 2413-2424.

Barcilon, A. I., 1967: Vortex decay above a stationary boundary. J. Fluid Mech., 27, 155-175.

Bluestein, H. B., W. P. Unruh, D. C. Dowell, T. A. Hutchinson, T. M. Crawford, A. C. Wood, and H. Stein, 1997: Doppler radar analysis of the Northfield, Texas tornado of 25 May 1994. Mon. Wea. Rev., 125, 212-230.

Brady, R. H., and E. J. Szoke, 1989: A case study of non-mesocyclone tornado development in Northeast Colorado: Similarities to waterspout formation. Mon. Wea. Rev., 117, 843-856.

Burggraf, O. R., K. Stewartson, and R. Belcher, 1971: Boundary layer induced by a potential vortex. Phys. Fluids, 14, 1821-1833. 
Carrier, G. F., 1971: Swirling flow boundary layers. J. Fluid Mech., 49, 133-144.

Church, C. R., J. T. Snow, G. L. Baker, and E. M. Agee, 1979: Characterisitics of tornado-like vortices as a function of swirl ratio: A laboratory investigation. J. Atmos. Sci., 36, 17551776.

Church, C. R., and J. T. Snow, 1993: Laboratory models of tornadoes. In The Tornado: its structure, dynamics, prediction, and hazards. C. Church, et. al., eds. American Geophysical Union, Washington, D. C.

Eliassen, A., 1971: On the Ekman layer in a circular vortex. J. Met. Soc. Japan, 49, 784.

Eliassen, A., and M. Lystad, 1977: The Ekman layer of a circular vortex. A numerical and theoretical study. Geophysica Norvegica, 31, 1-16.

Fiedler, B. H., 1993: Numerical simulation of axisymmetric tornadogenesis in forced convection. In The Tornado: its structure, dynamics, prediction, and hazards. C. Church, et. al., eds. American Geophysical Union, Washington, D. C.

1994: The thermodynamic speed limit and its violation in axisymmetric numerical simulations of tornado-like vortices. Atmosphere-Ocean, 32, 335-359.

1995: On modelling tornadoes in isolation from the parent storm. Atmosphere-

Ocean, 33, 501-512.

1998: Wind-speed limits in numerically simulated tornadoes with suction vor-

tices. Q. J. R. Meteorol. Soc., 123, 2377-2392. 
Fiedler, B. H., and R. Rotunno, 1986: A theory for the maximum windpseeds in tornado-like vortices. J. Atmos. Sci., 43, 2328-2340.

Gill, S. S., and J. Wurman, 1998: Wind field evolution of the Dimmit, TX and Rolla, KS tornadoes. 19th Conference on Severe Local Storms, Amer. Met. Soc., Boston.

Golden, J. H., 1974a: The life cycle of Florida Keys' waterspouts. I. J. Appl. Meteor., 13, 676691.

Golden, J. H., 1974b: Scale-interaction implications for the waterspout life cycle. J. Appl. Meteor., 13, 693-709.

Golden, J. H., and H. B. Bluestein, 1993: The NOAA-National Geographic Society waterspout expedition (1993). Bull. Amer. Met. Soc., 75, 2281-2288.

Grasso, L. D., and W. R. Cotton, 1995: Numerical simulation of a tornado vortex. J. Atmos. Sci., 52, 1192-1203.

Greenspan, H. P., 1990: The Theory of Rotating Fluids. Breukelen Press, Brookline, 325 pp.

Greenspan, H. P., and L. N. Howard, 1963: On a time dependent motion of a rotating fluid. J. Fluid Mech., 17, 385-404.

Howells, P. C., R. Rotunno, and R. K. Smith, 1988: A comparitive study of atmospheric and laboratory analogue numerical tornado-vortex models. Q. J. Roy. Met. Soc., 114, 801-822.

Kuo, H. L., 1971: Axisymmetric flows in the boundary layer of a maintained vortex. J. Atmos. Sci., 28, 20-41. 
Lee, B. D., and R. B. Wilhelmson, 1997a: The numerical simulation of non-supercell tornadogenesis. Part I: Initiation and evolution of pretornadic misocyclone circulations along a dry outflow boundary. J. Atmos. Sci., 54, 32-60.

Lee, B. D., and R. B. Wilhelmson, 1997b: The numerical simulation of non-supercell tornadogenesis. Part II: Evolution of a family of tornadoes along a weak outflow boundary. J. Atmos. Sci., 54, 2387-2415.

Lewellen, W. S., D. C., Lewellen, and R. Sykes, 1997: Large-eddy simulation of a tornado-'s interaction with the surface. J. Atmos. Sci., 54, 581-605.

Lewellen, D. C., W. S. Lewellen, and J. Xia, 2000: The influence of a local swirl ratio on tornado intensification near the surface. J. Atmos. Sci., 57, 527-544.

Nolan, D.S., and B. F. Farrell, 1999a: The structure and dynamics of tornado-like vortices. J. Atmos. Sci., 56, 2908-2936.

Nolan, D. S., and B. F. Farrell, 1999b: The intensification of two-dimensional swirling flows by stochastic, asymmetric forcing. J. Atmos. Sci., 56, 3937-3962.

Rasmussen, E. N., J. M. Straka, R. Davies-Jones, C. A. Doswell, F. H. Carr, M. D. Eilts, and D. R. MacGorman, 1994: Verification of the origin of rotation in tornadoes experiment: VORTEX. Bull. Amer. Meteor. Soc., 75, 995-1006.

Rotunno, R., 1977: Numerical simulation of a laboratory vortex. J. Atmos. Sci., 34, 1942-1956. 1979: A study in tornado-like vortex dynamics. J. Atmos. Sci., 36, 140-155. 
Simpson, J., B. R. Morton, M. C. McCumber, and R. S. Penc, 1986: Observations and mechanisms of GATE waterspouts. J. Atmos. Sci., 43, 753-782.

Sinclair, P. C., 1969: General characteristics of dust devils. J. Appl. Met., 8, 32-45. 1973: The lower structure of dust devils. J. Atmos. Sci., 30, 1599-1619.

Trapp, R. J., 1999: Observations of nontornadic low-level mesocyclones and attendant tornadogenesis failure during VORTEX. Mon. Wea. Rev., 127, 1693-1705.

Trapp, R. J., and B. H. Fiedler, 1995: Tornado-like vortexgenesis in a simplified numerical model. J. Atmos. Sci., 52, 3757-3778.

Trapp, R. J., and R. Davies-Jones, 1997: Tornadogenesis with and without a dynamic pipe effect. J. Atmos. Sci., 54, 113-132.

Walko, R. L., and R. Gall, 1986: Some effects of momentum diffusion on axisymmetric vortices. J. Atmos. Sci., 43, 2137-2148.

Wakimoto, R. M., and N. T. Atkins, 1996: Observations on the origins of rotation: The Newcastle tornado during VORTEX 1994. Mon. Wea. Rev., 124, 384-407.

Wakimoto, R. M., C. Liu, and H. Cai, 1998: The Garden City, Kansas storm during VORTEX 95. Part I: Overview of the storm's life cycle and mesocyclogenesis. Mon. Wea. Rev., 126, $372-392$.

Wakimoto, R. M., and C. Liu, 1998: The Garden City, Kansas storm during VORTEX 95. Part II: The wall cloud and tornado. Mon. Wea. Rev., 126, 393-408. 
Wakimoto, R. M., and J. F. Wilson, 1989: Non-supercell tornadoes. Mon. Wea. Rev., 117, 11131140.

Ward, N. B., 1972: The exploration of certain features of tornado dynamics using a laboratory model. J. Atmos. Sci., 29, 1194-1204.

Wicker, L. W., and R. B. Wilhelmson, 1995: Simulation and analysis of tornado development and decay within a three-dimensional supercell thunderstorm. J. Atmos. Sci., 52, 2675-2703.

Wurman, Joshua, 1998: Preliminary results from the ROTATE-98 tornado study. 19th Conference on Severe Local Storms, Amer. Met. Soc., Boston.

Wurman, J., J. M. Straka, and E. N. Rasmussen, 1996: Fine-scale doppler radar observations of tornadoes. Science, 272, 1774-1777. 
Table 1 Summary of parameters for solid-body rotation simulations.

\begin{tabular}{|c|c|c|c|c|c|c|c|c|c|}
\hline $\begin{array}{l}\text { Simulation } \\
\text { Name }\end{array}$ & $\begin{array}{l}\text { Domain } \\
\text { Height }\end{array}$ & $\begin{array}{l}\text { Base } \\
\text { Grid }\end{array}$ & $\begin{array}{l}\text { Max. } \\
\text { ref. } \\
\text { lvs. }\end{array}$ & $\Omega$ & $v$ & $C_{b}$ & $z_{\text {forc }}$ & $\sigma_{h}$ & $\sigma_{v}$ \\
\hline F93FULL & 1.0 & $256 \times 128$ & 0 & 0.2 & 0.0005 & 1.264 & 0.5 & 0.2236 & 0.2236 \\
\hline F93AMR & 1.0 & $64 \times 32$ & 2 & 0.2 & 0.0005 & 1.264 & 0.5 & 0.2236 & 0.2236 \\
\hline F93ZDBL & 2.0 & $64 \times 64$ & 3 & 0.2 & 0.0005 & 1.264 & 0.5 & 0.2236 & 0.2236 \\
\hline HIBUBL & 2.0 & $64 \times 64$ & 3 & 0.2 & 0.0005 & 1.264 & 1.0 & 0.2236 & 0.2236 \\
\hline $\begin{array}{l}\text { TALL- } \\
\text { BUBL }\end{array}$ & 2.0 & $64 \times 64$ & 3 & 0.2 & 0.0005 & 0.632 & 1.0 & 0.2236 & 0.4472 \\
\hline WIDBUBL & 2.0 & $64 \times 64$ & 3 & 0.2 & 0.0005 & 1.264 & 1.0 & 0.4472 & 0.2236 \\
\hline $\begin{array}{c}\text { WID- } \\
\text { BUBL } \Omega .05\end{array}$ & 2.0 & $64 \times 64$ & 3 & 0.05 & 0.0005 & 1.264 & 1.0 & 0.4472 & 0.2236 \\
\hline $\begin{array}{c}\text { F93 } \\
\text { DBLU }\end{array}$ & 1.0 & $64 \times 32$ & 3 & 0.2 & 0.0005 & 5.07 & 0.5 & 0.2236 & 0.2236 \\
\hline $\begin{array}{l}\text { HISWIRL } \\
\text { 2CELL }\end{array}$ & 1.0 & $128 \times 64$ & 3 & 0.5 & 0.0005 & 1.264 & 0.5 & 0.2236 & 0.2236 \\
\hline $\begin{array}{l}\text { LOW } \\
\text { SWIRL } \\
\text { 2CELL }\end{array}$ & 1.0 & $128 \times 64$ & 3 & 0.2 & 0.0002 & 1.264 & 0.5 & 0.2236 & 0.2236 \\
\hline $\begin{array}{c}\text { LOW } \\
\text { SWIRL } \\
\text { DVJ }\end{array}$ & 1.0 & $128 \times 64$ & 3 & 0.08 & 0.0002 & 1.264 & 0.5 & 0.2236 & 0.2236 \\
\hline $\begin{array}{l}\text { LOWEST } \\
\text { VISC }\end{array}$ & 1.0 & $256 \times 128$ & 3 & 0.1 & 0.0001 & 1.264 & 0.5 & 0.4472 & 0.2236 \\
\hline
\end{tabular}


Table 2 Summary of statisitical results for solid-body rotation simulations.

\begin{tabular}{|c|c|c|c|c|c|c|c|}
\hline Simulation & $\overline{C_{v}}$ & $\overline{\mathrm{RMW}}$ & $\overline{\mathrm{ZMW}}$ & $\overline{A_{V}}$ & $\left(\overline{\left(A_{V}-\overline{A_{V}}\right)^{2}}\right)^{\frac{1}{2}}$ & $C_{\mathrm{ZMW}}$ & $C_{\text {RMW }}$ \\
\hline F93FULL & 0.7318 & 0.1205 & 0.0725 & 1.7400 & 0.4810 & 1.450 & 0.370 \\
\hline F93AMR & 0.7320 & 0.1218 & 0.0722 & 1.7509 & 0.4262 & 1.444 & 0.374 \\
\hline F93ZDBL & 0.7444 & 0.1124 & 0.0726 & 1.6188 & 0.5677 & 1.452 & 0.345 \\
\hline HIBUBL & 0.7384 & 0.1153 & 0.0683 & 1.7039 & 0.2517 & 1.366 & 0.354 \\
\hline $\begin{array}{l}\text { TALL } \\
\text { BUBL }\end{array}$ & 0.7360 & 0.1121 & 0.0717 & 1.5891 & 0.2732 & 1.434 & 0.344 \\
\hline WIDBUBL & 0.7609 & 0.2745 & 0.0716 & 3.8854 & 0.7808 & 1.432 & 0.354 \\
\hline $\begin{array}{c}\text { WID } \\
\text { BUBL } 2.05\end{array}$ & 0.7838 & 0.1846 & 0.1370 & 1.4085 & 0.4797 & 1.370 & 0.283 \\
\hline F93DBLU & 0.7734 & 0.1079 & 0.0710 & 1.6031 & 0.4597 & 1.420 & 0.331 \\
\hline $\begin{array}{l}\text { HISWIRL } \\
\text { 2CELL }\end{array}$ & 0.7067 & 0.1400 & 0.0475 & 2.9733 & 0.3941 & 1.502 & 0.383 \\
\hline $\begin{array}{c}\text { LOW } \\
\text { SWIRL } \\
\text { 2CELL }\end{array}$ & 0.8129 & 0.1196 & 0.0454 & 2.7966 & 0.8140 & 1.436 & 0.327 \\
\hline $\begin{array}{c}\text { LOW } \\
\text { SWIRL } \\
\text { DVJ }\end{array}$ & 0.7970 & 0.0991 & 0.0694 & 1.4864 & 0.3521 & 1.388 & 0.304 \\
\hline $\begin{array}{l}\text { LOWEST } \\
\text { VISC }\end{array}$ & 1.04 & 0.158 & 0.0420 & 4.06 & 0.9677 & 1.323 & 0.182 \\
\hline
\end{tabular}


Table 3 Summary of Tornado-Scale Simulation Parameters.

\begin{tabular}{c|c|c|c|c|c|c|c|c}
\hline $\begin{array}{c}\text { Simulation } \\
\text { Name }\end{array}$ & $\begin{array}{c}\text { Base } \\
\text { Grid }\end{array}$ & $\begin{array}{c}\text { Max. } \\
\text { ref. } \\
\text { lvs. }\end{array}$ & $\begin{array}{c}r_{\max } \\
(\mathrm{m})\end{array}$ & $\begin{array}{c}V^{*} \\
\left(\mathrm{~ms}^{-1}\right)\end{array}$ & $\begin{array}{c}v \\
\left(\mathrm{~m}^{2} \mathrm{~s}^{-1}\right)\end{array}$ & $\begin{array}{c}z_{f o r c} \\
(\mathrm{~m})\end{array}$ & $\begin{array}{c}\sigma_{h} \\
(\mathrm{~m})\end{array}$ & $\begin{array}{c}\sigma_{v} \\
(\mathrm{~m})\end{array}$ \\
\hline TORNADO & $256 \times 256$ & 3 & 2000 & 15 & 40 & 4000 & 2000 & 2000 \\
\hline $\begin{array}{c}\text { TORNADO } \\
\text { HIVORT }\end{array}$ & $256 \times 256$ & 3 & 2000 & 30 & 40 & 4000 & 2000 & 2000 \\
\hline $\begin{array}{c}\text { TORNADO } \\
\text { HIVISC }\end{array}$ & $256 \times 256$ & 3 & 2000 & 15 & 80 & 4000 & 2000 & 2000 \\
\hline $\begin{array}{c}\text { TORNADO } \\
\text { WIDE }\end{array}$ & $256 \times 256$ & 3 & 2000 & 15 & 40 & 4000 & 3000 & 2000 \\
\hline
\end{tabular}


Table 4 Summary of Statistical Results for Tornado-Scale Simulations.

\begin{tabular}{c|c|c|c|c|c|c|c}
\hline Simulation & $\overline{C_{v}}$ & $\begin{array}{c}\overline{\mathrm{RMW}} \\
(\mathrm{m})\end{array}$ & $\begin{array}{c}\overline{\mathrm{ZMW}} \\
(\mathrm{m})\end{array}$ & $\overline{A_{V}}$ & $\left(\overline{\left(A_{V}-\overline{A_{V}}\right)^{2}}\right)^{\frac{1}{2}}$ & $C_{\mathrm{ZMW}}$ & $C_{\mathrm{RMW}}$ \\
\hline TORNADO & 0.83 & 188.2 & 116.2 & 1.66 & 0.383 & 1.59 & 0.0409 \\
\hline $\begin{array}{c}\text { TORNADO } \\
\text { HIVORT }\end{array}$ & 1.11 & 289.44 & 102.2 & 3.07 & 1.23 & 1.98 & 0.0577 \\
\hline $\begin{array}{c}\text { TORNADO } \\
\text { HIVISC }\end{array}$ & 0.56 & 204.0 & 175.6 & 1.18 & 0.29 & 1.70 & 0.0484 \\
\hline $\begin{array}{c}\text { TORNADO } \\
\text { WIDE }\end{array}$ & 0.59 & 173.2 & 154.0 & 1.24 & 0.664 & 1.41 & 0.0251 \\
\hline
\end{tabular}




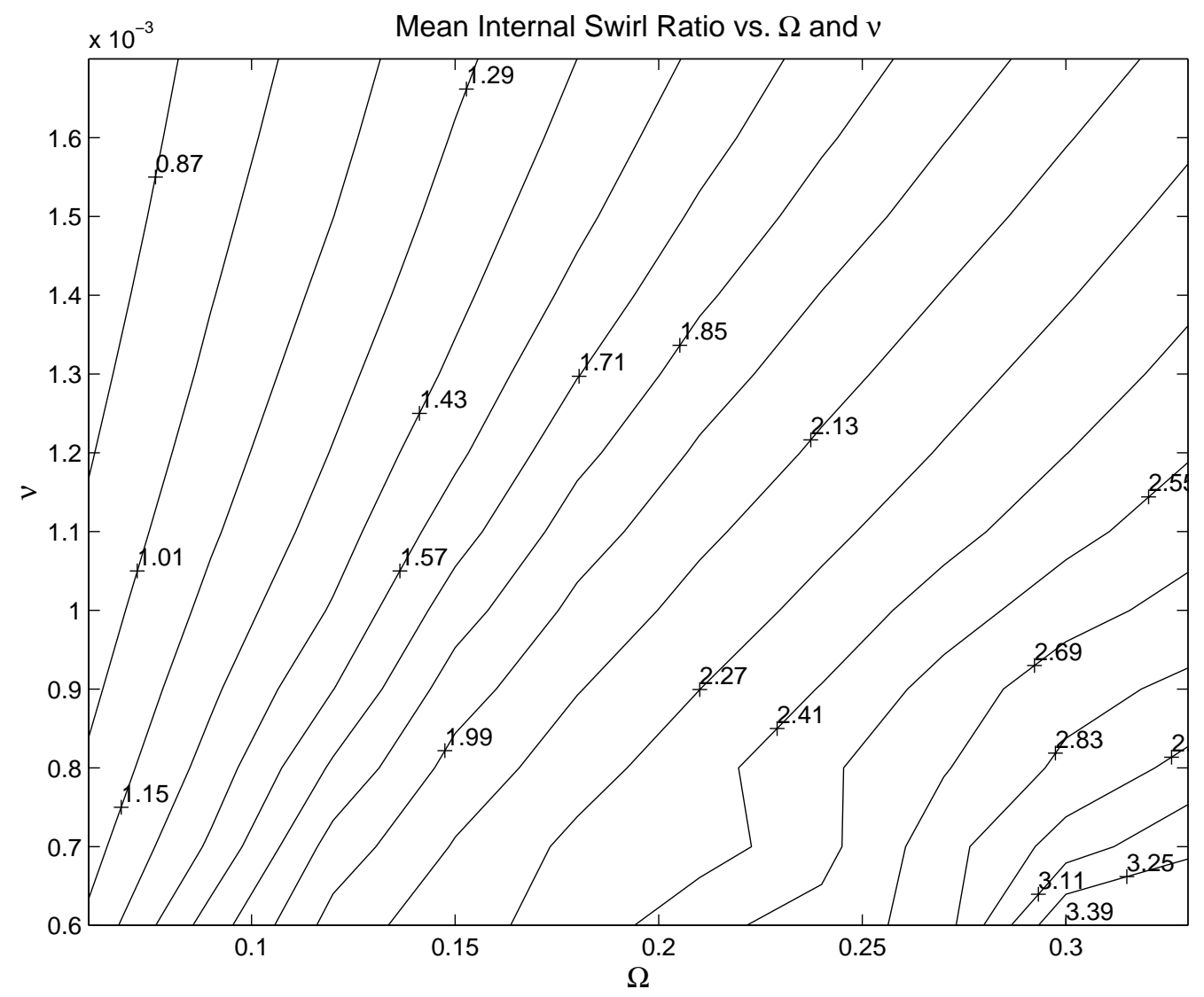

Fig. 1 Contour plot of the mean value of the internal swirl ratio as a function of the domain rotation rate $\Omega$ and the eddy viscosity $v$. 


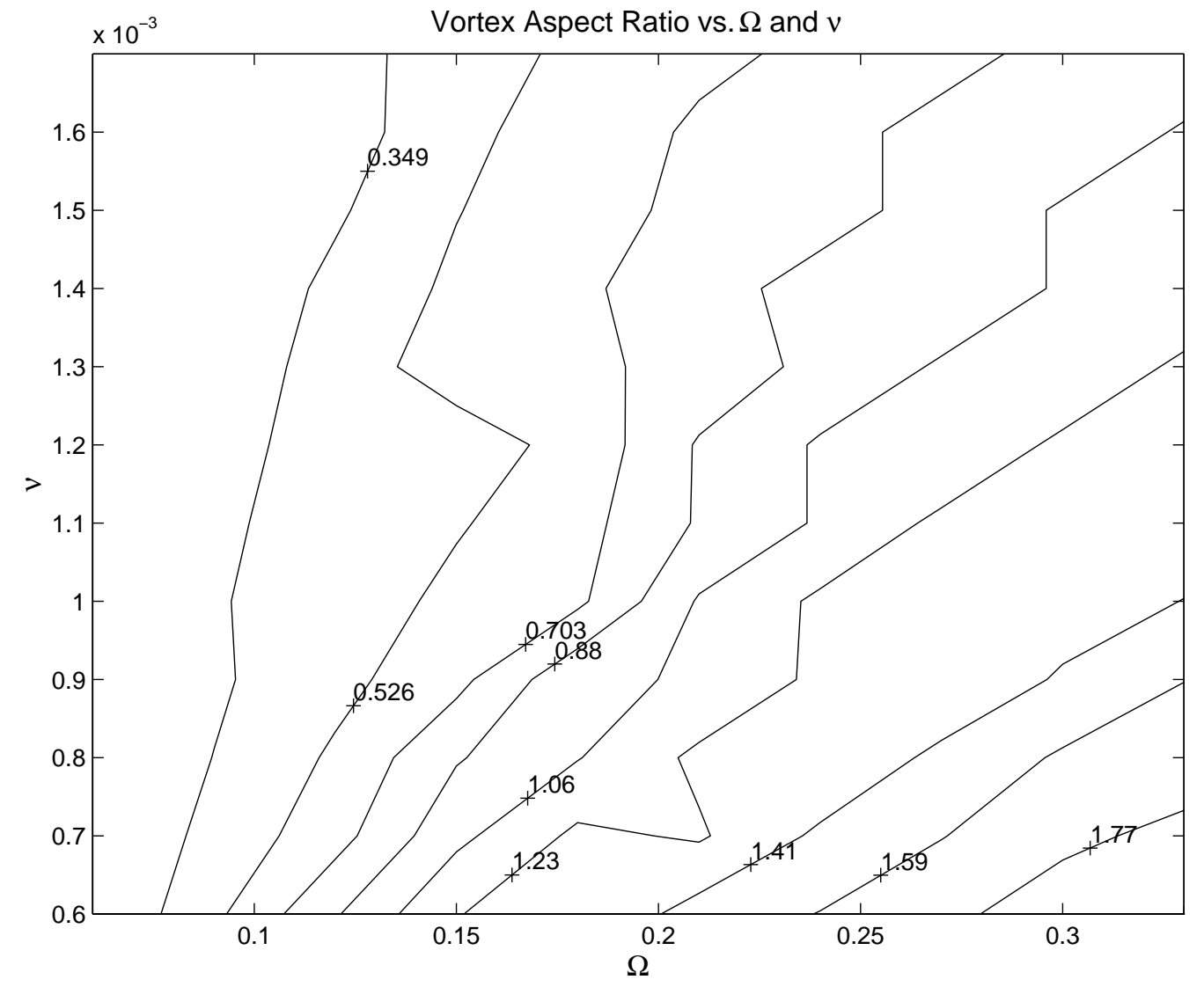

Fig. 2 Contour plot of the mean vortex aspect ratio as a function of the domain rotation rate and the eddy viscosity. 


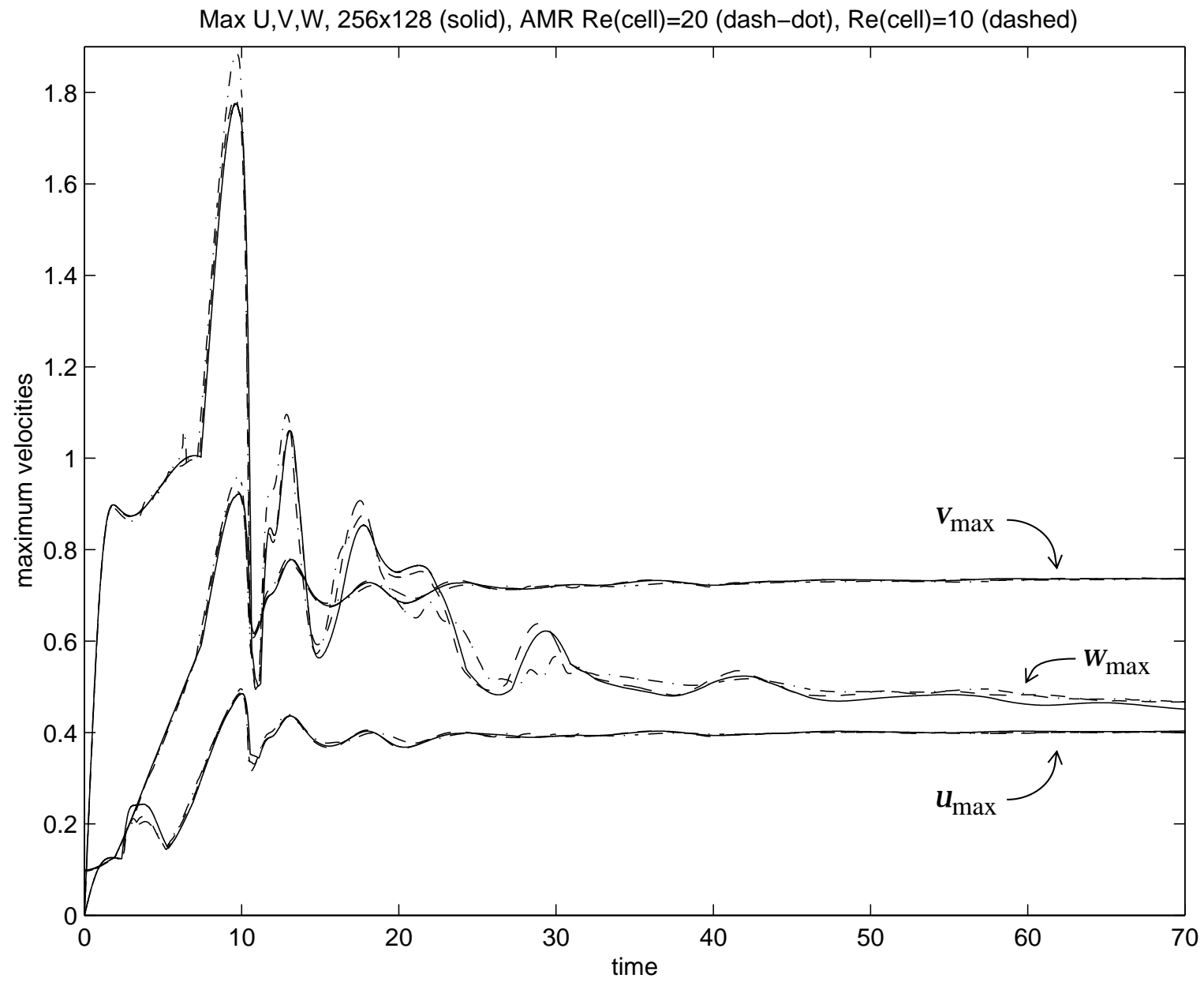

Fig. 3 Maximum vertical, azimuthal, and negative horizontal velocities for the F93 simulation with 256x128 gridpoints (solid), the AMR simulation with $R e_{c e l l}<20$ (dash-dot), and the AMR simulation with $R e_{c e l l}<10$ (dashed). 
a)

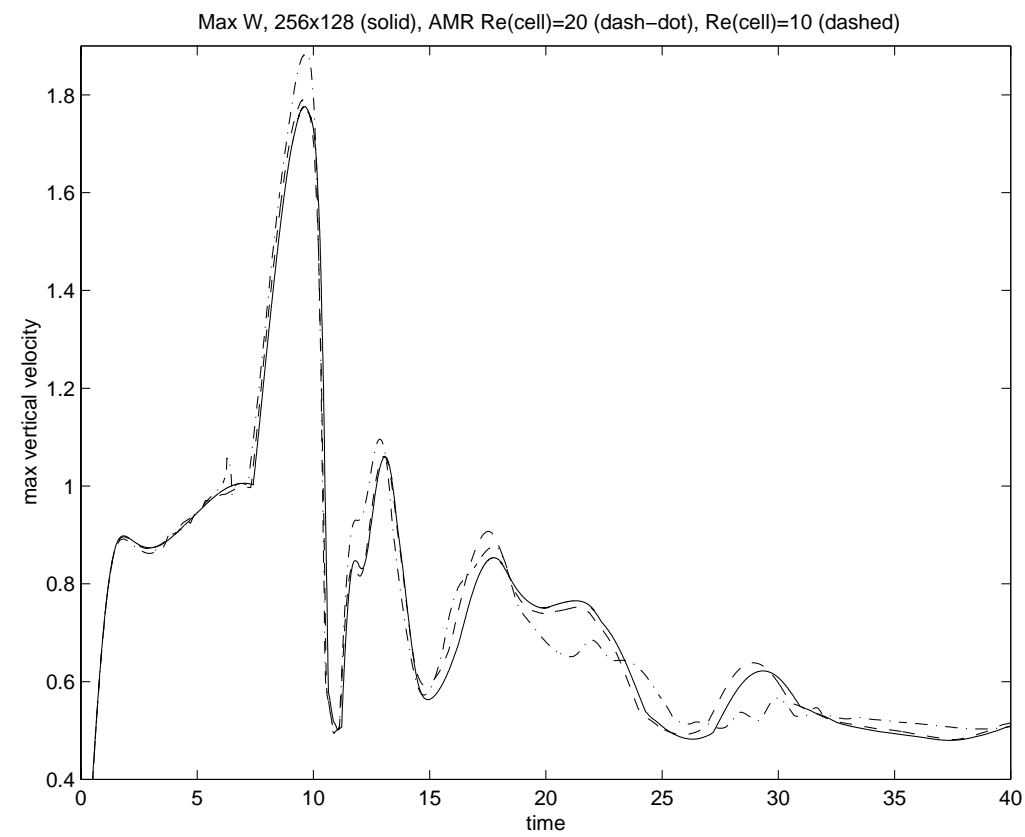

b)

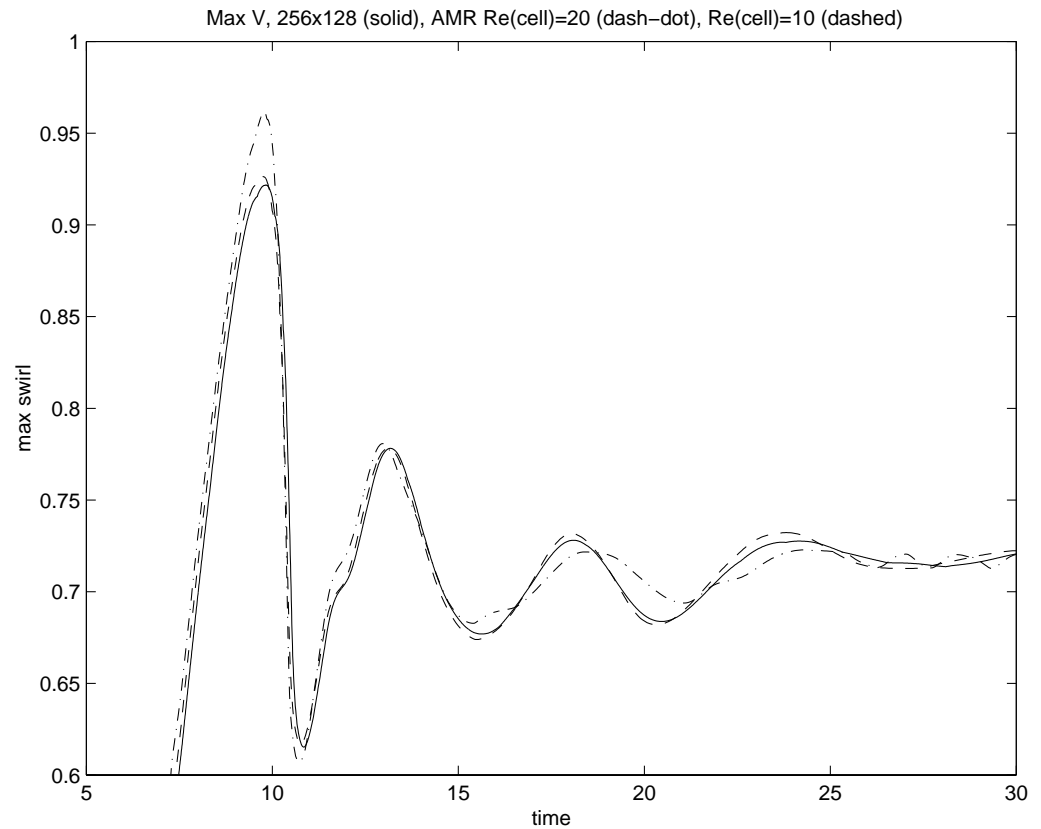

Fig. 4 Same as previous but for a) the maximum vertical velocities only, and b) for the maximum azimuthal velocities only. 
a)

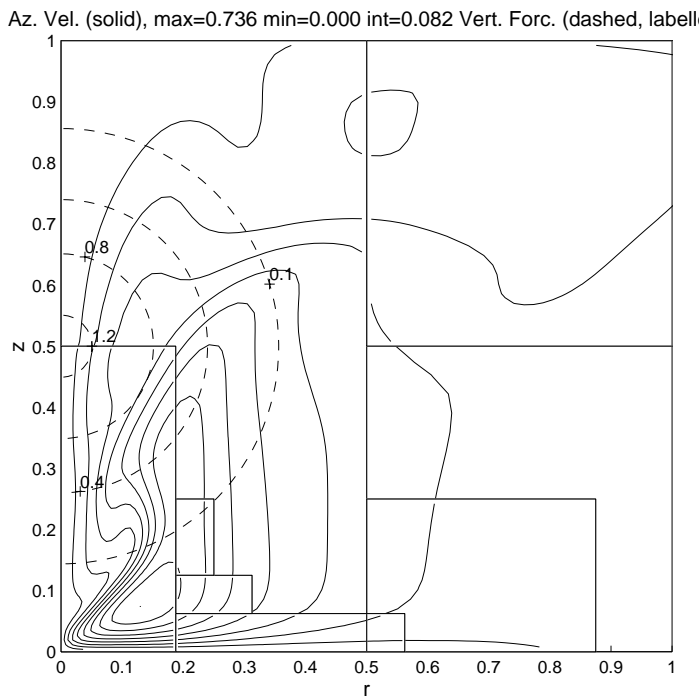

c)

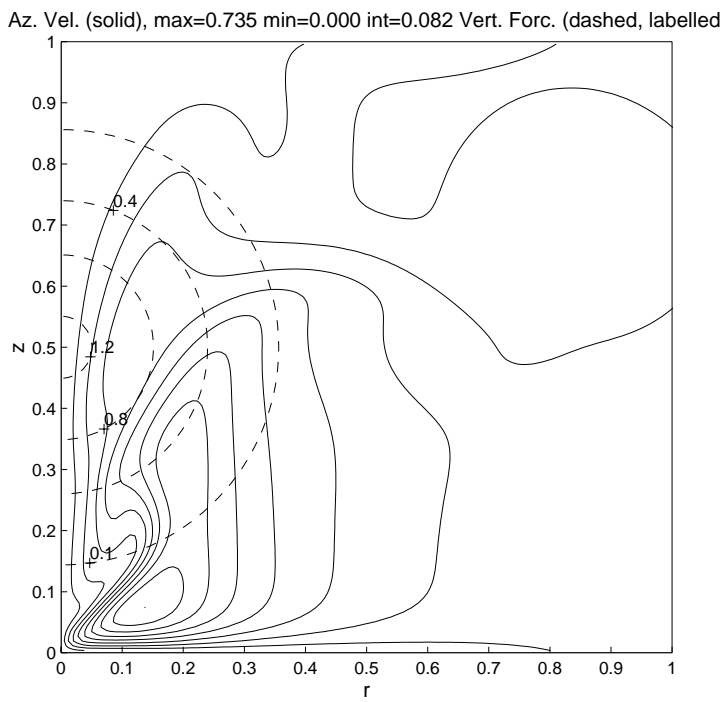

b)

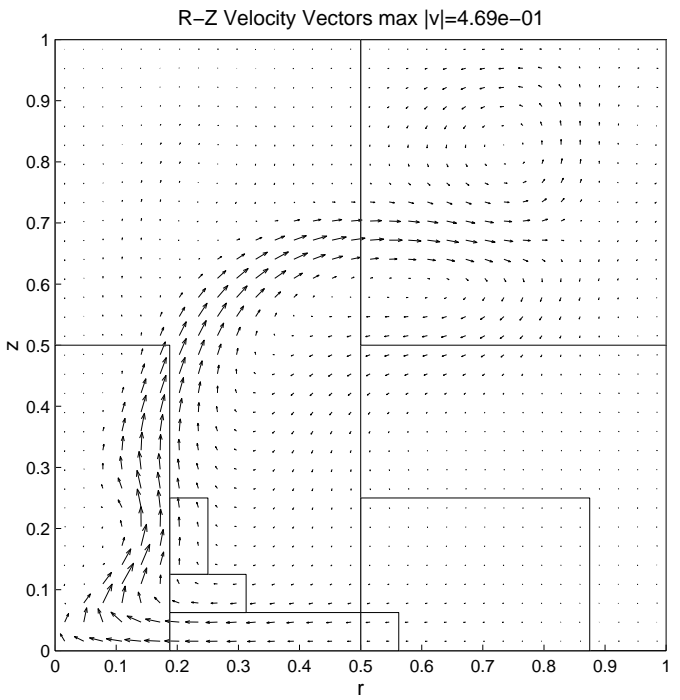

d)

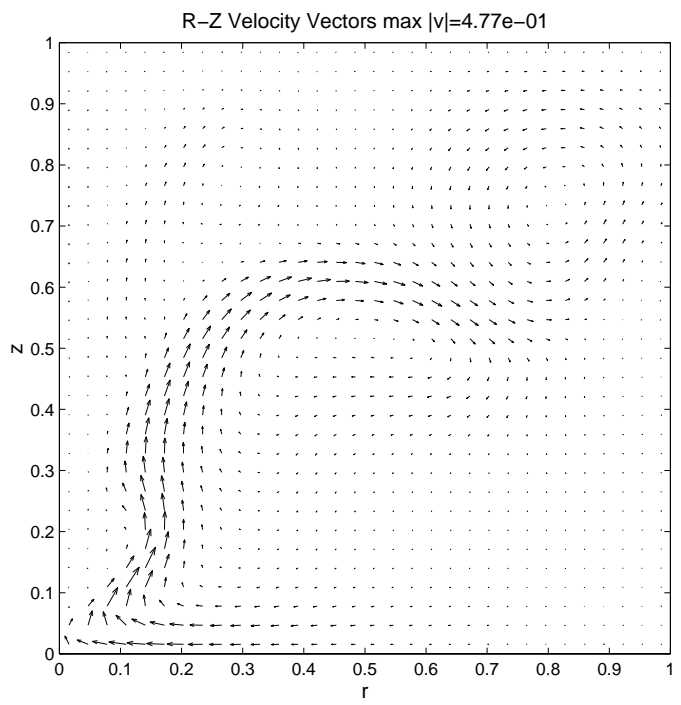

Fig. 5 A direct comparison velocity fields for the F93 simulations at $t=100.0$, a) azimuthal velocities and vertical forcing, with AMR; b) meridional velocity vectors on the base grid, with AMR; c) azimuthal velocities and vertical forcing with full resolution throughout the domain; d) meridional velocity vectors with full resolution - here the data has been interpolated onto a $64 \times 32$ grid. Plots are restricted to the part of the domain where $0 \leq r \leq 1$. 
a)

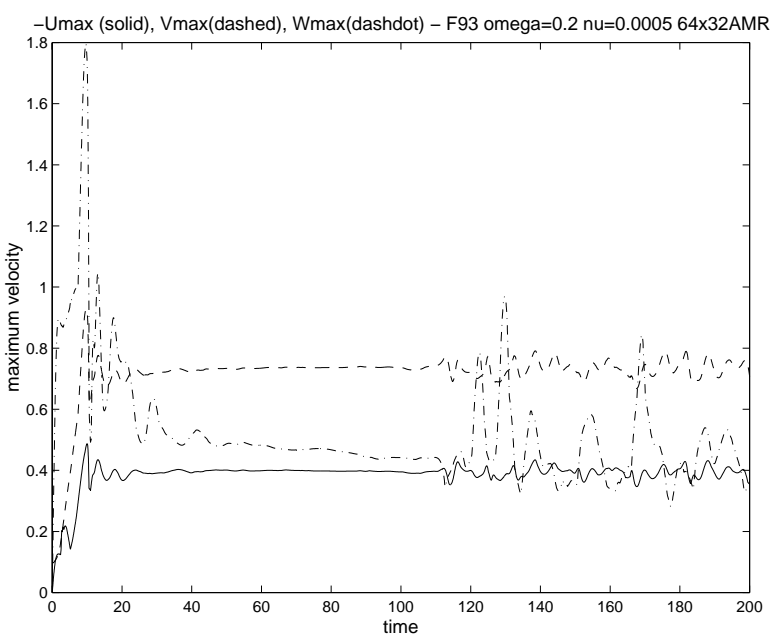

c)

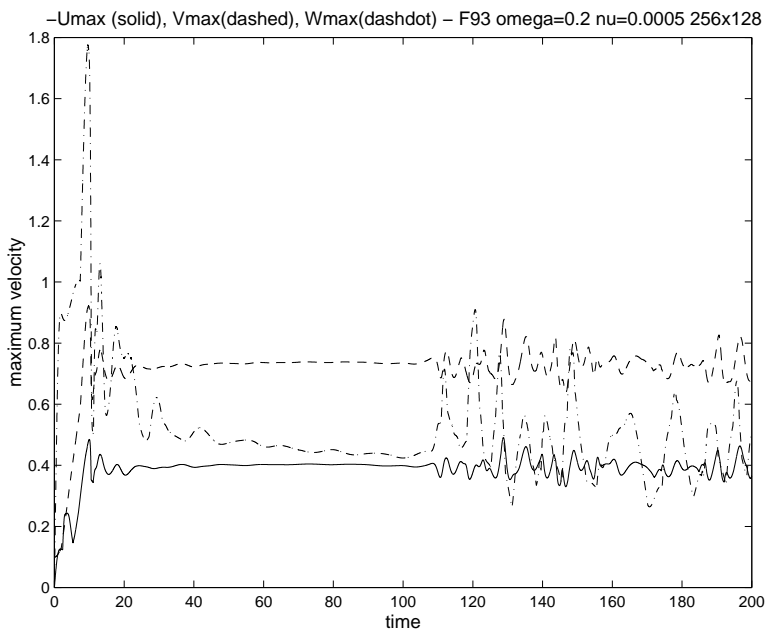

b)

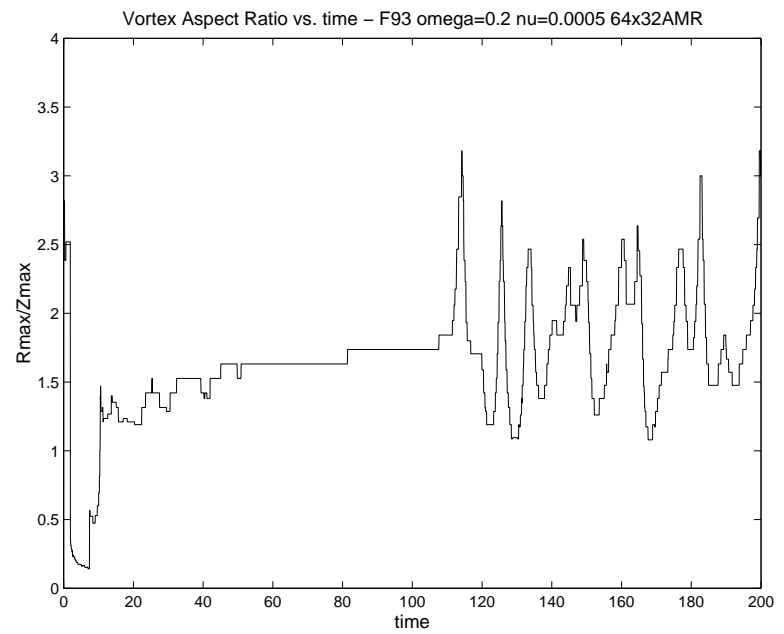

d)

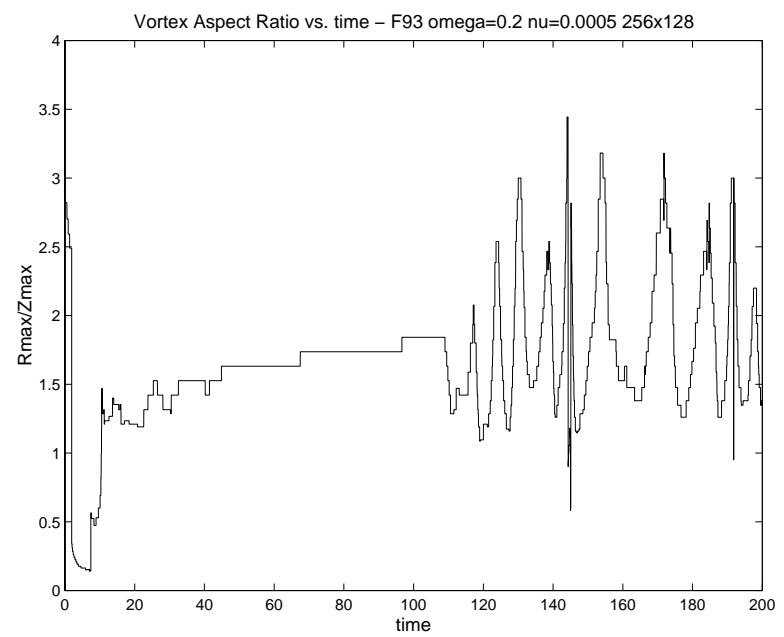

Fig. 6 Maximum velocities and vortex aspect ratios as a function of time during the AMR and full resolution F93 simulations: a) maximum azimuthal velocities (dashed), vertical velocities (dash-dot), and inward radial velocities (solid), AMR; b) vortex aspect ratio, AMR; c) maximum velocities, full resolution; d) vortex aspect ratio, full resolution. 
a)
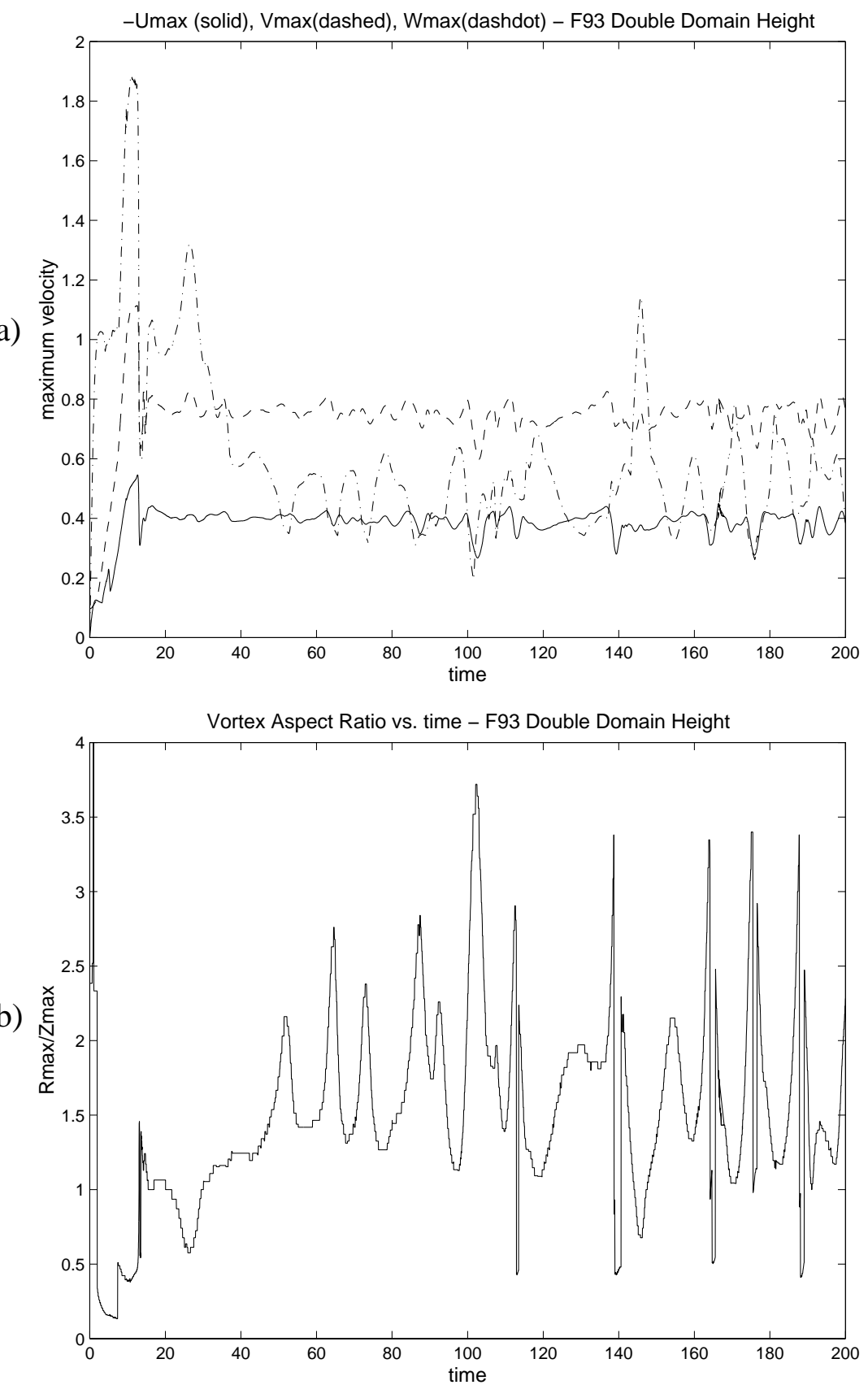

Fig. 7 Results for the F93ZDBL simulation: a) maximum azimuthal velocities (dashed), vertical velocities (dash-dot), and inward radial velocities (solid); b) vortex aspect ratio. 
a)

Az. Vel. (solid), $\max =0.760 \mathrm{~min}=0.000 \mathrm{int}=0.084$ Vert. Forc. (dashed, labelled)

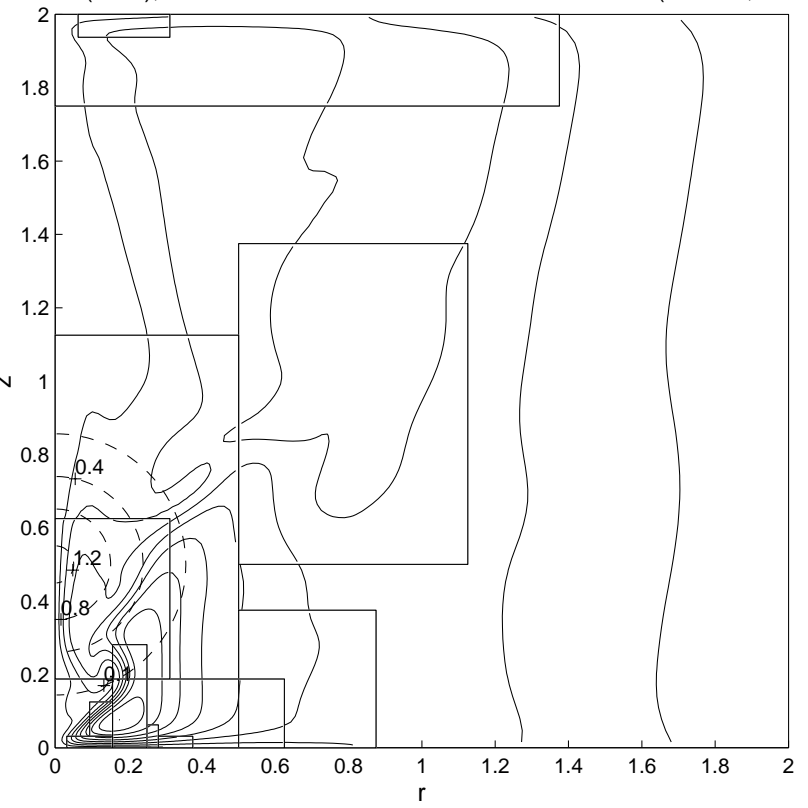

b)

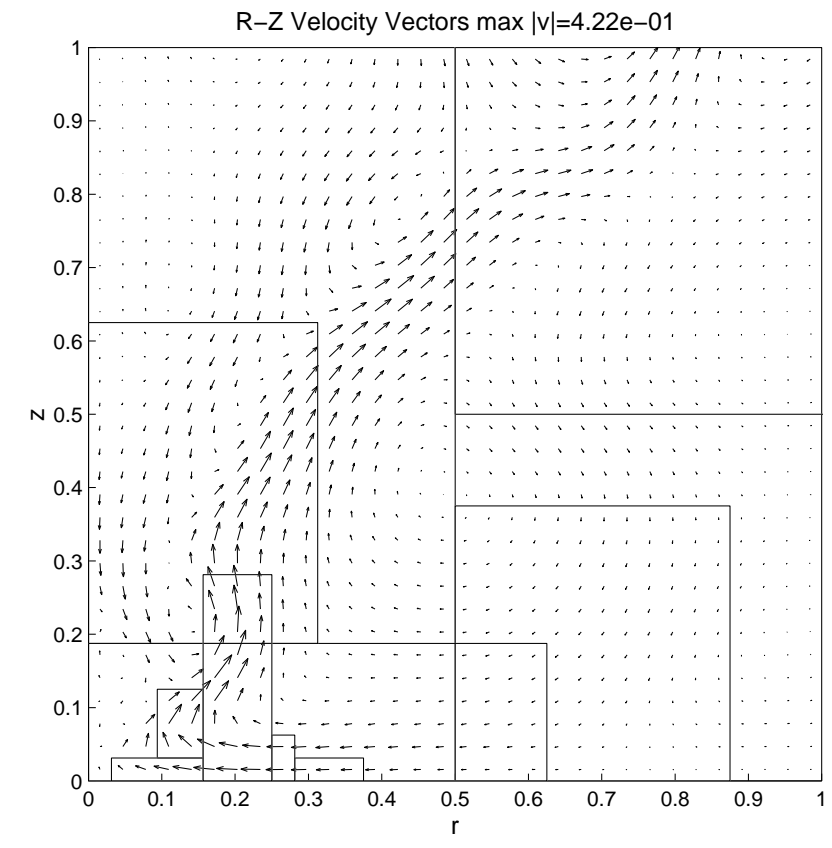

Fig. 8 Azimuthal and vertical velocity fields at $t=200$ for the F93ZDBL simulation: a) azimuthal velcoities and vertical forcing; b) meridional velocity vectors on the base grid in the region $0<r<1.0,0<z<1.0$. 
a)

Az. Vel. (solid), $\max =0.690 \mathrm{~min}=0.000 \mathrm{int}=0.077$ Vert. Forc. (dashed, labelled)

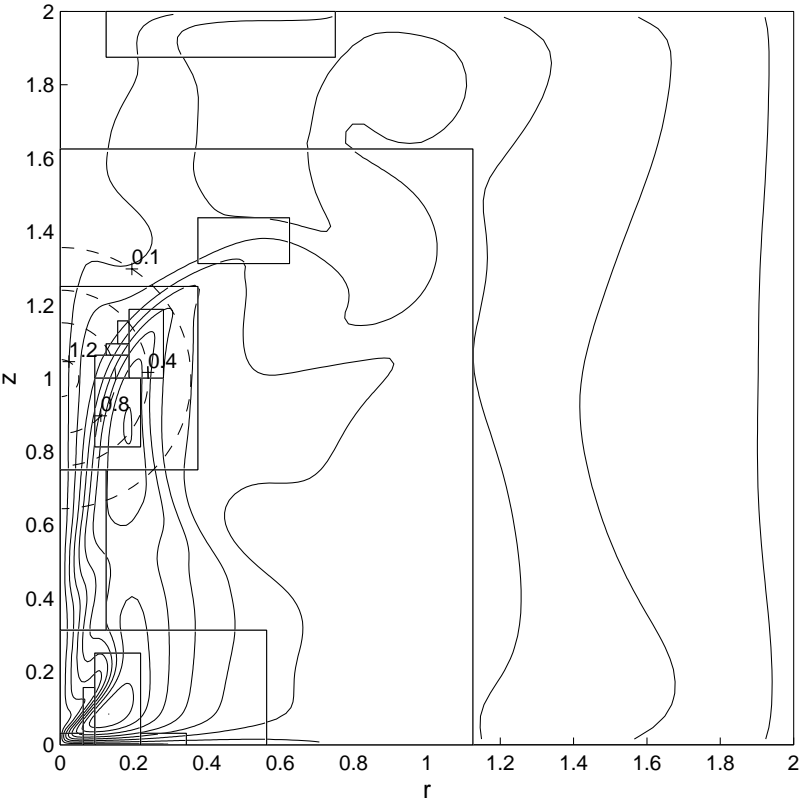

R-Z Velocity Vectors max $|\mathrm{V}|=4.42 \mathrm{e}-01$

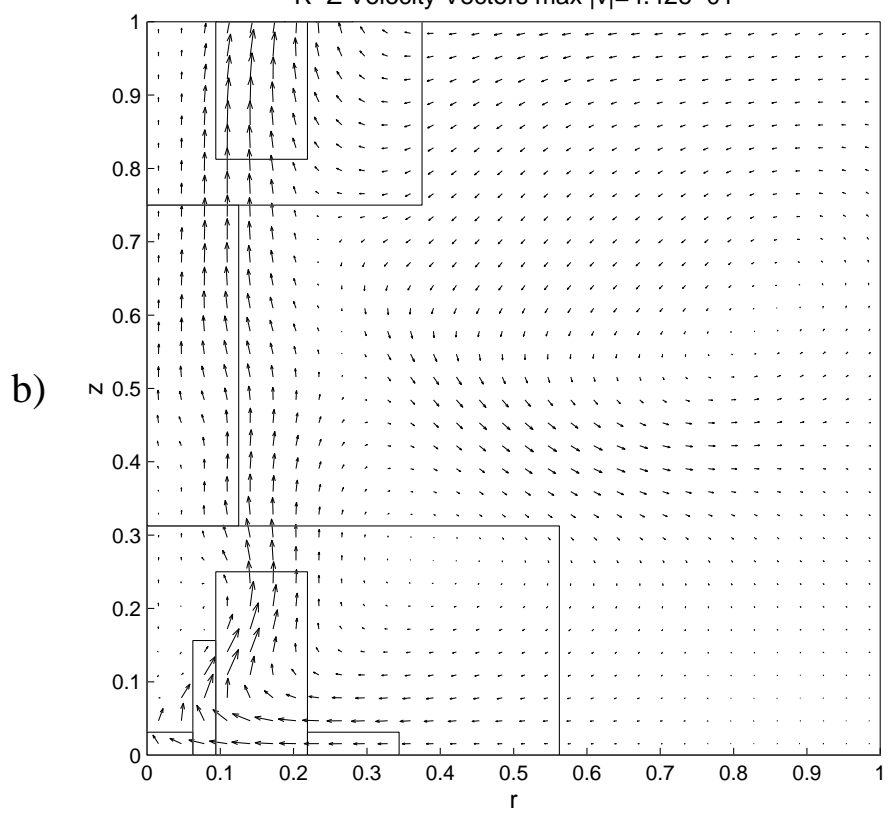

Fig. 9 Azimuthal and vertical velocity fields at $t=200$ for the HIBUBL simulation: a) azimuthal velcoities and vertical forcing; $b$ ) meridional velocity vectors on the base grid in the region $0<r<1.0,0<z<1.0$. 
a)

Az. Vel. (solid), $\max =0.751 \mathrm{~min}=0.000 \mathrm{int}=0.083$ Vert. Forc. (dashed, labelled)

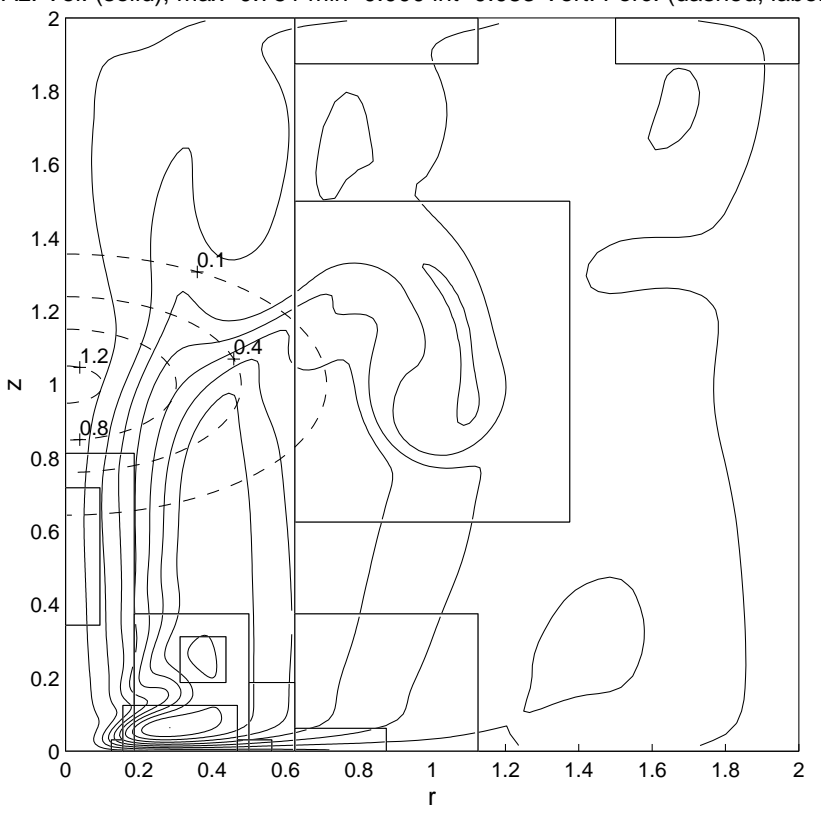

b)

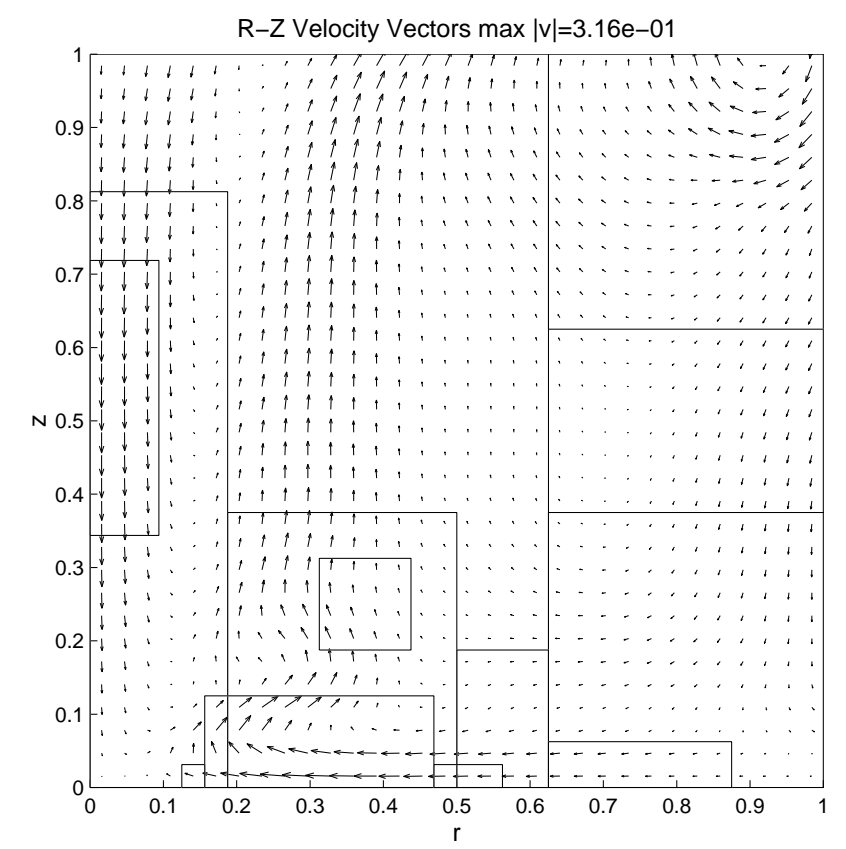

Fig. 10 Azimuthal and vertical velocities at $t=100$ for the WIDBUBL simulation: a) azimuthal velocities and vertical forcing; b) meridional velocity vectors on the base grid in the region $0<r<1.0,0<z<1.0$. 
a)

Az. Vel. (solid), $\max =0.714 \mathrm{~min}=0.000 \mathrm{int}=0.079$ Vert. Forc. (dashed, labelled)

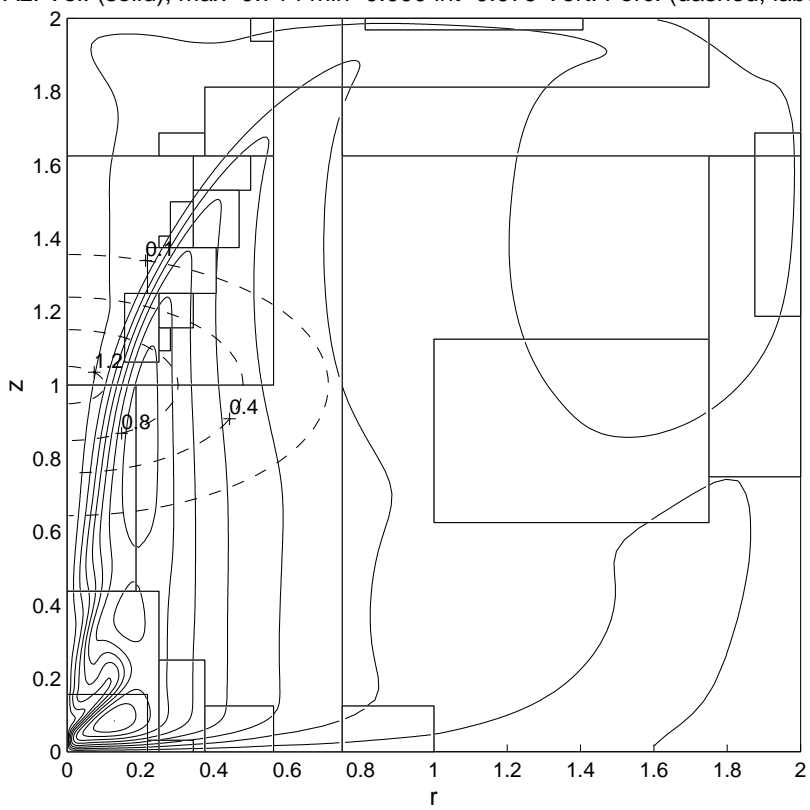

R-Z Velocity Vectors max $|v|=6.57 e-01$

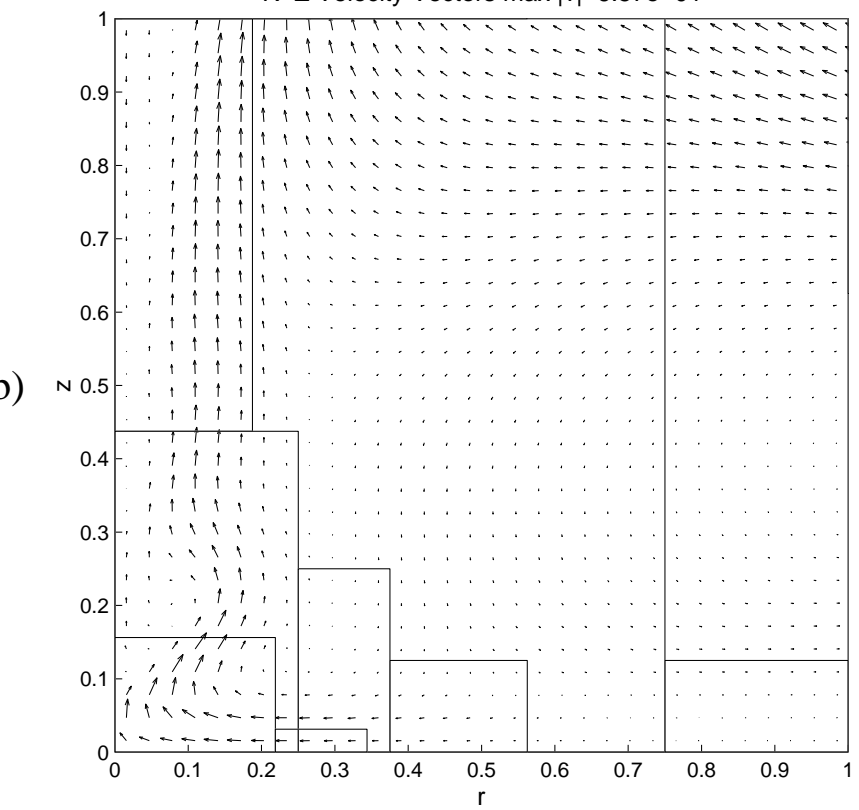

Fig. 11 Azimuthal and vertical velocities at $t=100$ for the WIDBUBL $\Omega 05$ simulation: a) azimuthal velocities and vertical forcing; $b$ ) meridional velocity vectors on the base grid in the region $0<r<1.0,0<z<1.0$. 
a)

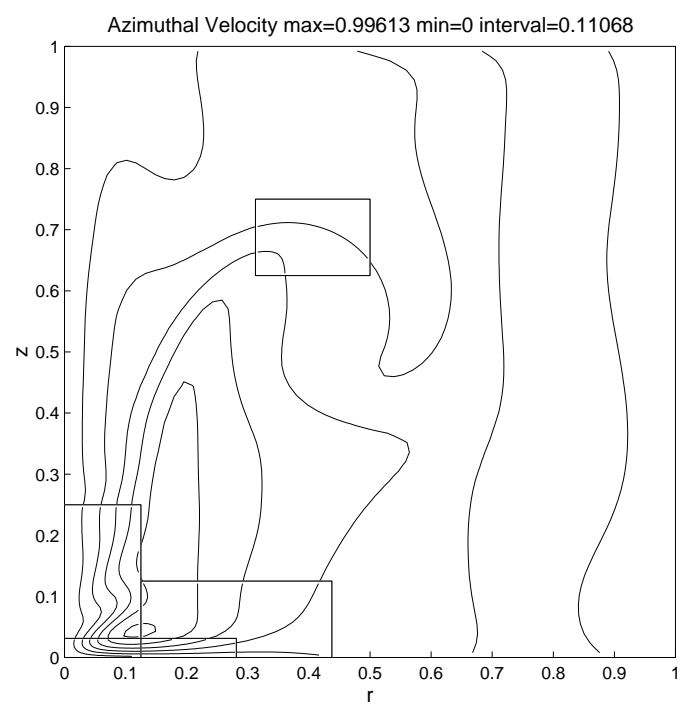

c)

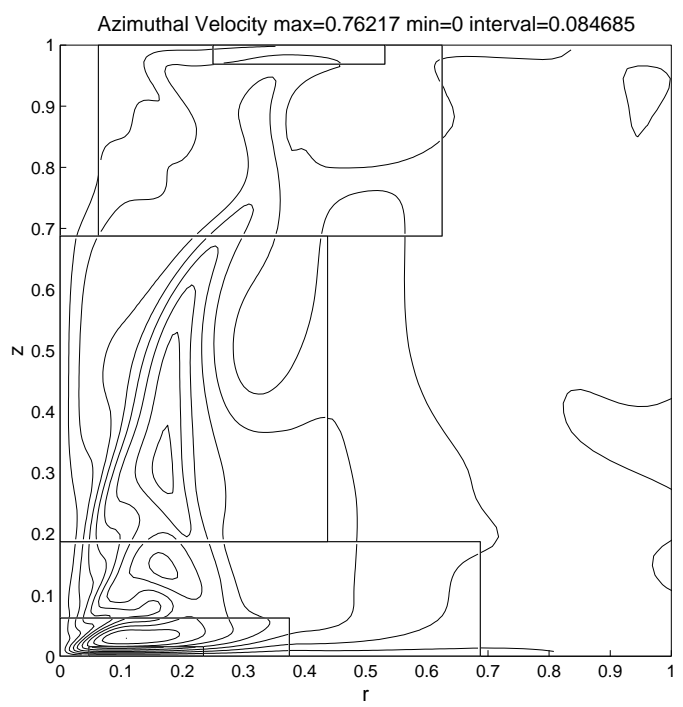

b)

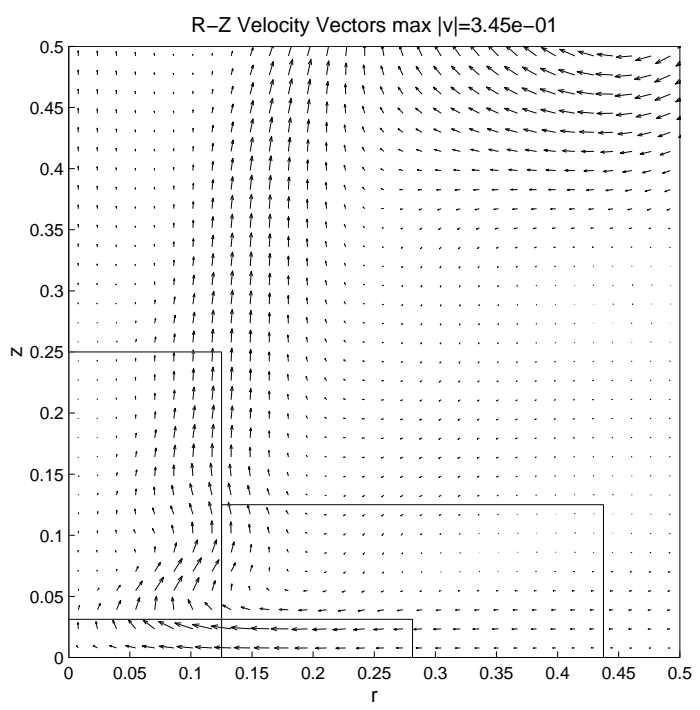

d)

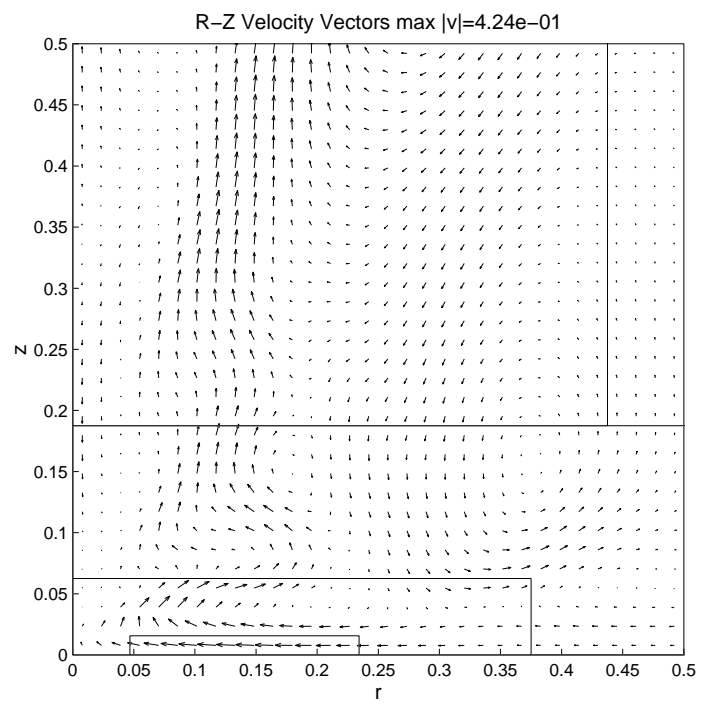

Fig. 12 Azimuthal and vertical velocities at $t=200$ for the HISWIRL2CELL and LOWSWIRL2CELL simulations and : a) azimuthal velocities, HISWIRL2CELL; b) meridional velocity vectors on hte base grid in the region $0<r<0.5,0<z<0.5$. HISWIRL2CELL; c) azimuthal velocities, LOWSWIRL2CELL, d) meridional velocity vectors on the base grid in the region $0<r<0.5,0<z<0.5$, LOWSWIRL2CELL The vertical forcings are the same as in Fig. 5. 


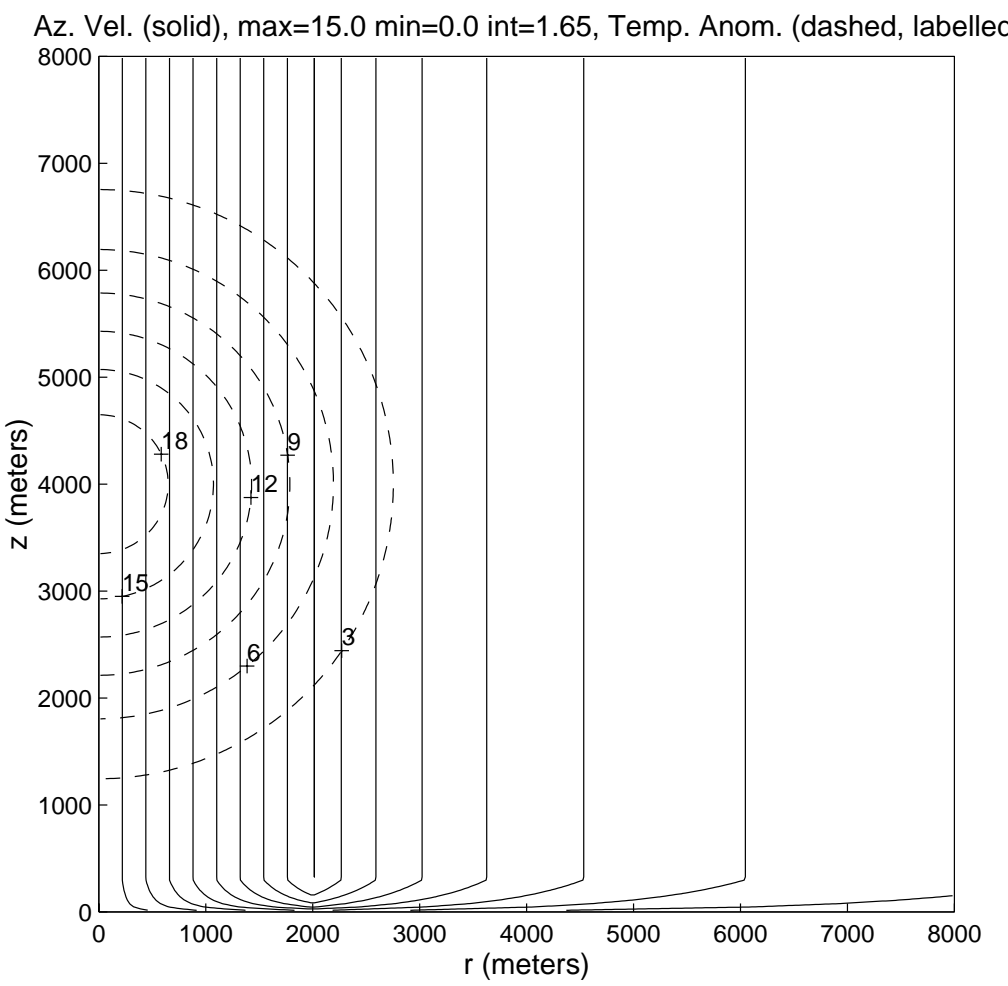

Fig. 13 Contour plots of the initial azimuthal velocity field (solid) and the effective temperature anomaly (dashed) associated with the convective forcing for the tornado-scale simulations. 
a)

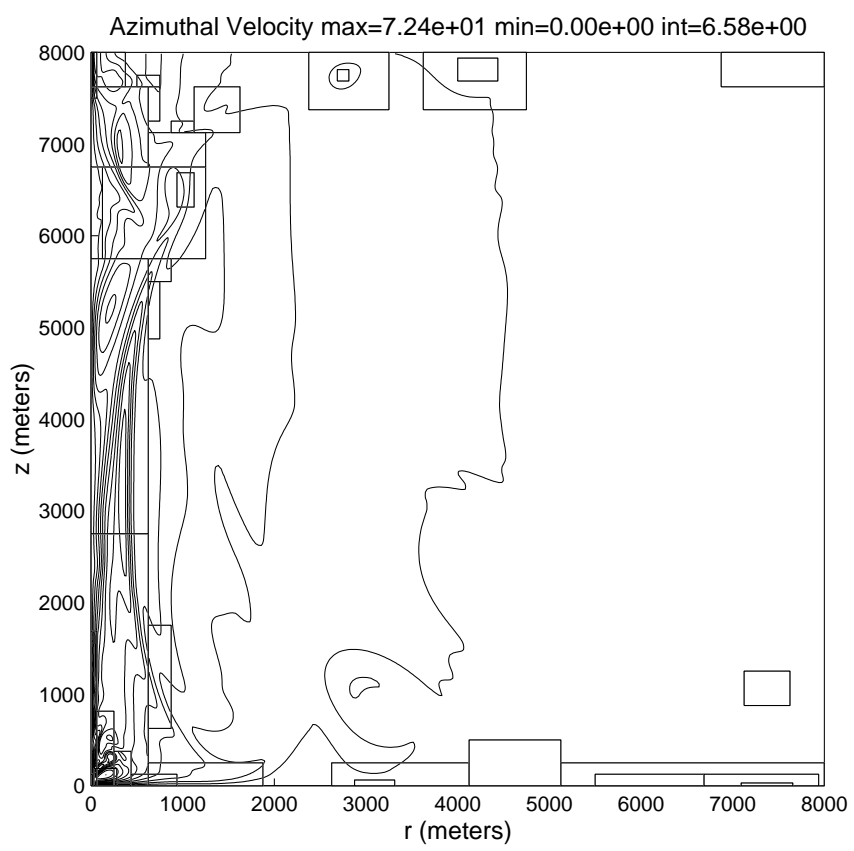

b)

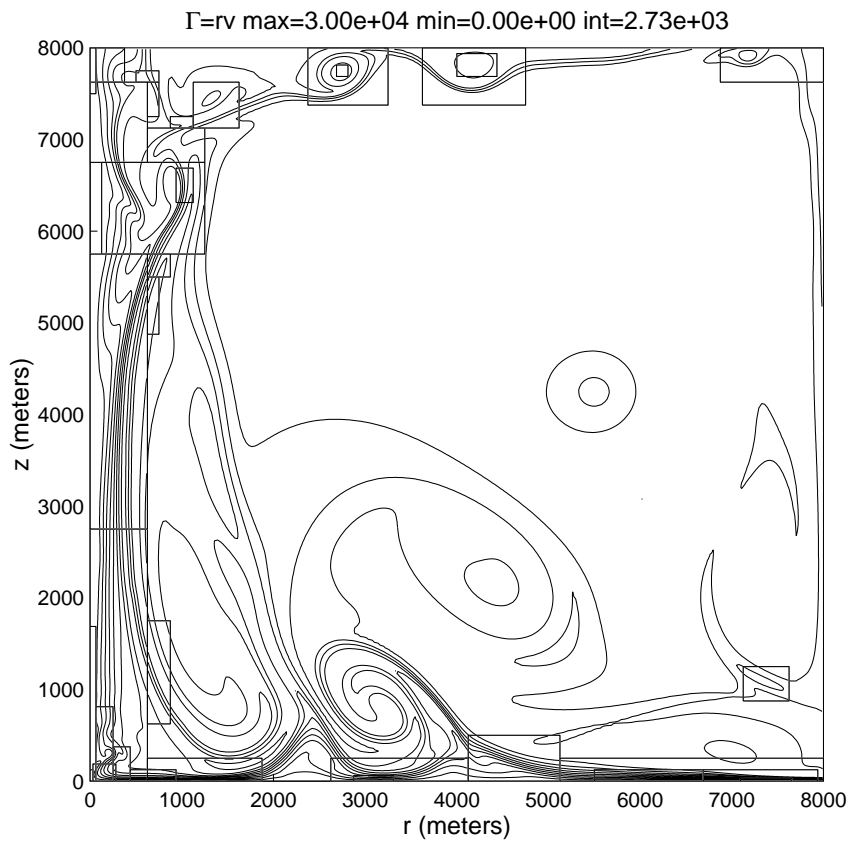

Fig. 14 Contour plots of the a) azimuthal velocity field, and b) the circulation $\Gamma$ in the tornado-scale simulation at $t=1616 \mathrm{~s}$. 

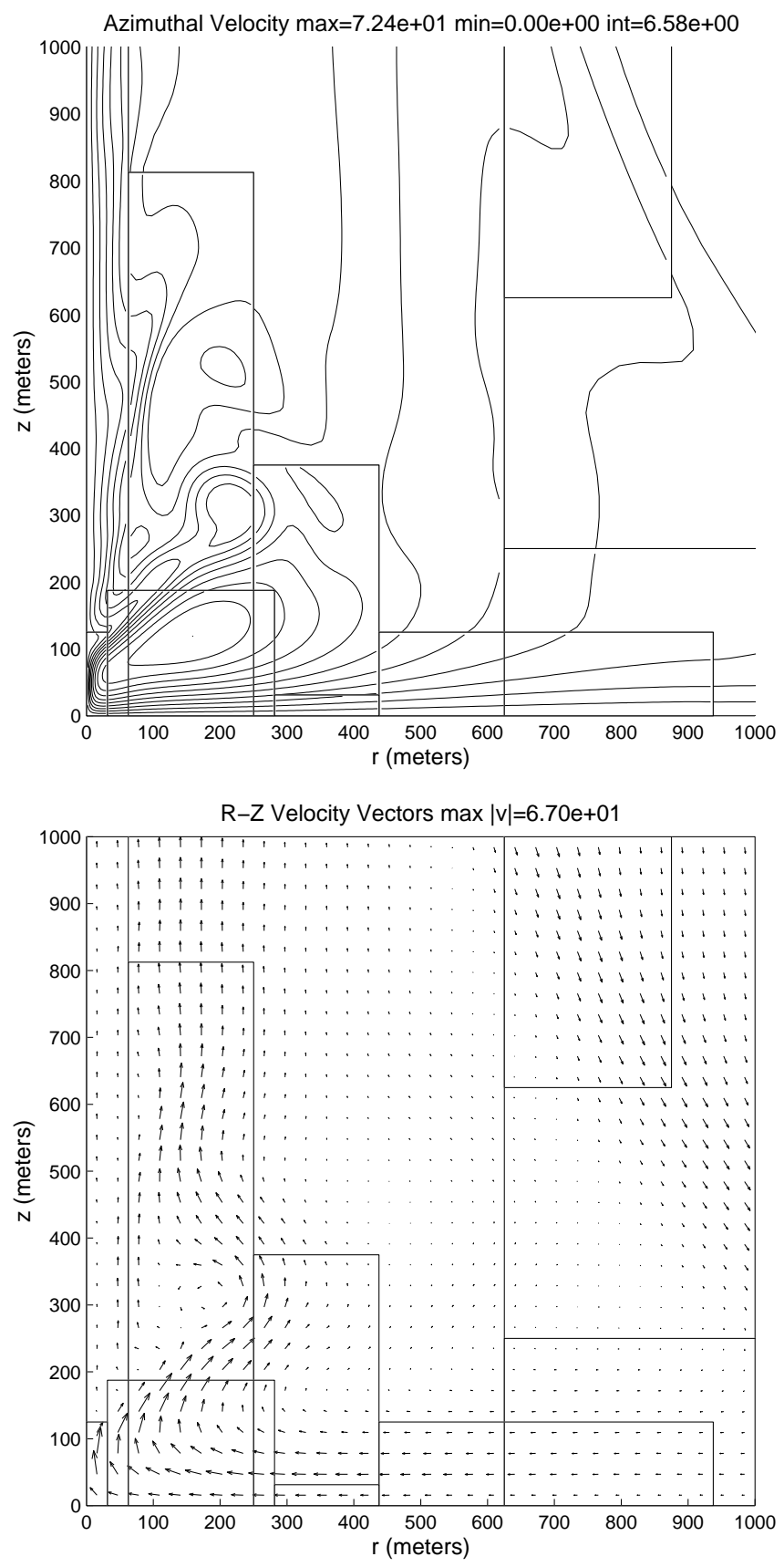

Fig. 15 Close-up of the a) azimuthal velocity field and b) meridional velocity vectors in the low-level vortex generated in the tornado-scale simulation at $t=1616 \mathrm{~s}$. Only the data from the base grid is used in the vector plot. 


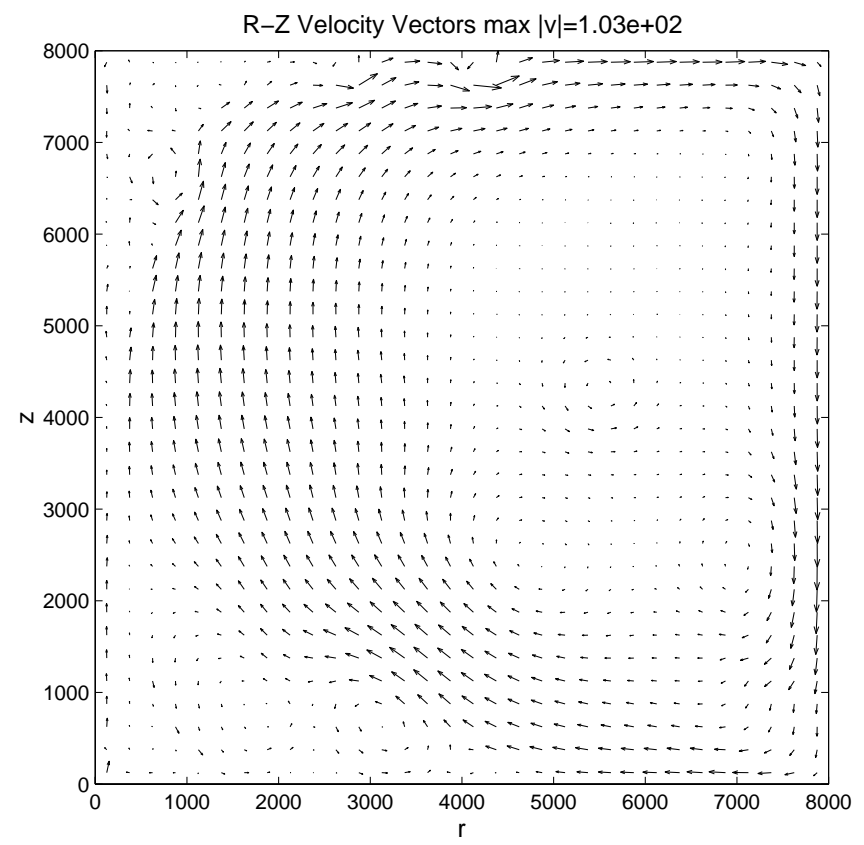

Fig. 16 Meridional velocity vectors at $t=1616$ in the tornado-scale simulation. The data is derived from the base grid, interpolated onto a 32x32 grid. 
a)

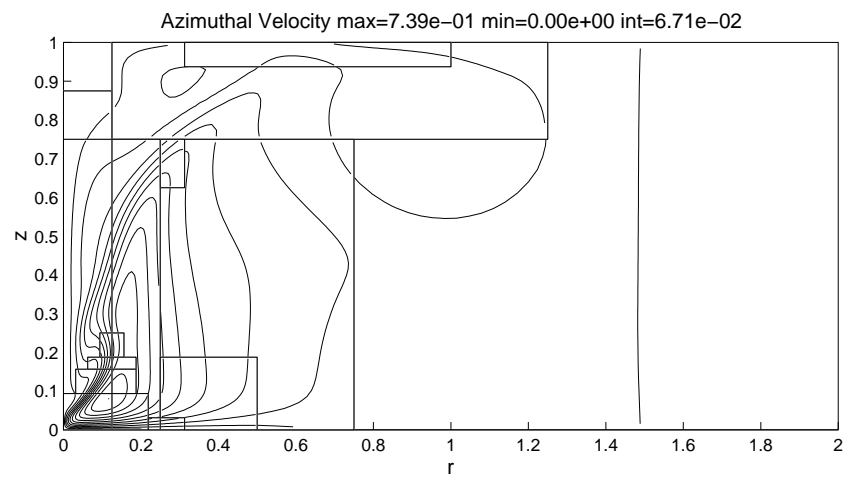

b)
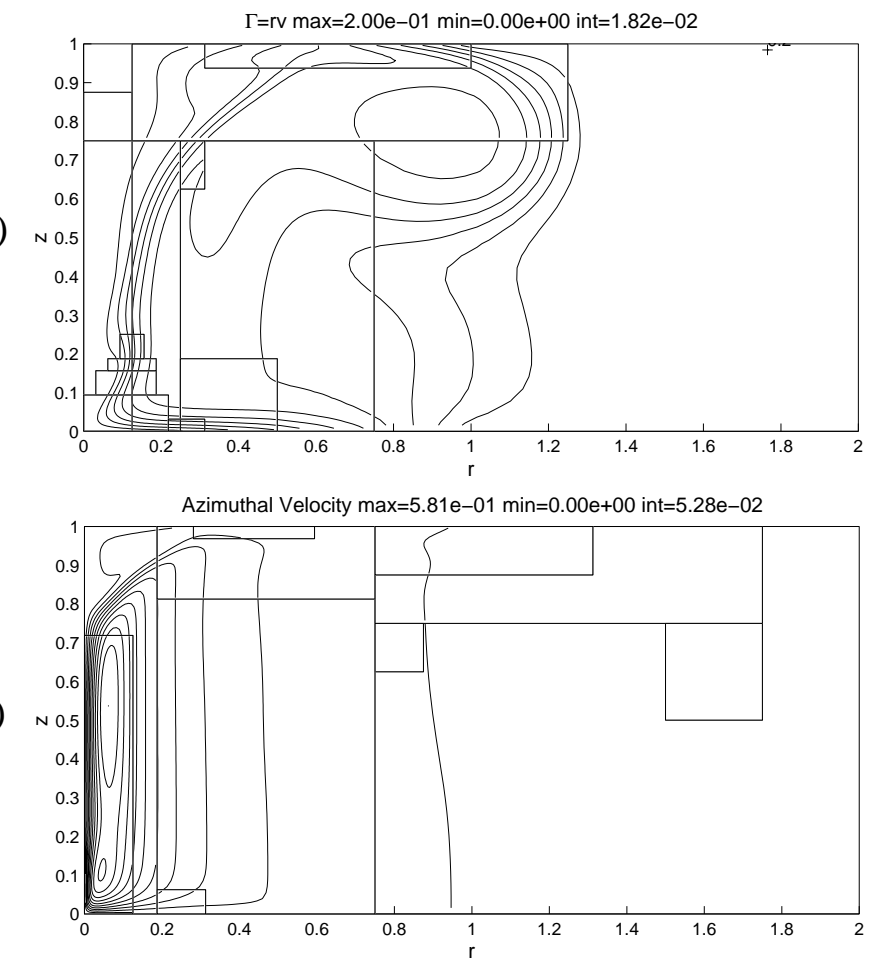

c)

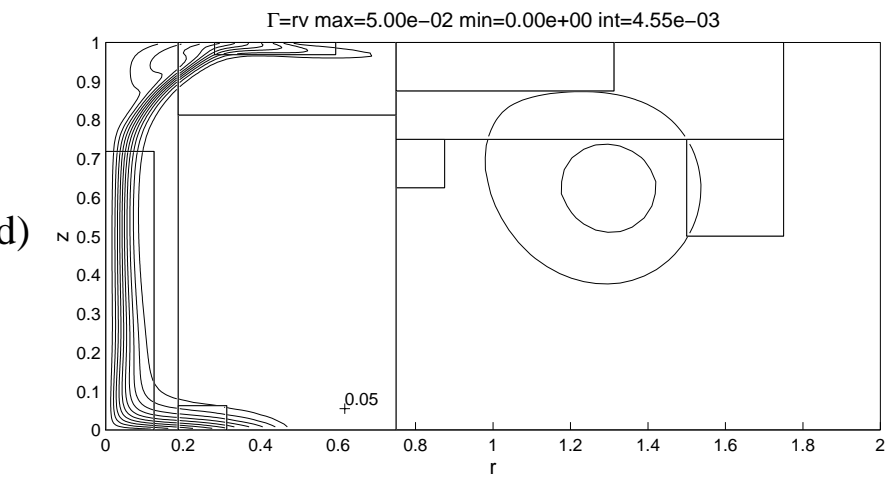

Fig. 17 Snapshots of azimuthal velocity and circulation in F93-type simulations with finite circulation in the far field: a) azimuthal velocity, $r_{\max }=1.0$; b) circulation, $r_{\max }=1.0 ; \mathrm{c}$ ) azimuthal velocity, $r_{\max }=0.5 ;$ d) circulation, $r_{\max }=0.5$. 